\title{
Gradhiva
}

GRADHIV

Revue d'anthropologie et d'histoire des arts

\section{La lune de Saturne et le «nous » œcuménique. Entre astrobiologie et anthropologie}

An Icy Moon of Saturn and the Ecumenical 'We'. Between Astrobiology and Anthropology

Istvan Praet

Traducteur : Camille Joseph

\section{OpenEdition}

\section{Journals}

Édition électronique

URL : http://journals.openedition.org/gradhiva/3061

DOI : 10.4000/gradhiva.3061

ISSN : 1760-849X

Éditeur

Musée du quai Branly Jacques Chirac

Édition imprimée

Date de publication : 1 octobre 2015

Pagination : 136-167

ISBN : 978-2-35744-092-0

ISSN : 0764-8928

Référence électronique

Istvan Praet, «La lune de Saturne et le «nous » œcuménique. Entre astrobiologie et anthropologie », Gradhiva [En ligne], 22 | 2015, mis en ligne le 01 octobre 2018, consulté le 20 avril 2019. URL : http:// journals.openedition.org/gradhiva/3061 ; DOI : 10.4000/gradhiva.3061

(c) musée du quai Branly 



\section{La lune de Saturne \\ et le «nous» cecuménique \\ Entre astrobiologie et anthropologie}

par Istvan Praet

Cet article aborde l'exploration spatiale d'un point de vue anthropologique en privilégiant avant tout le domaine de l'astrobiologie et, plus particulièrement, les recherches menées aujourd'hui sur Titan, la plus grande lune de Saturne. Titan constitue l'un des sujets favoris des astrobiologistes: cette lune possède en effet toute une série de caractéristiques, parmi lesquelles une atmosphère très dense, qui lui confèrent un étrange air de famille avec la Terre. Mais comment en est-on venu à établir cette soi-disant familiarité? Pour y répondre, je me propose d'étudier ce que j'appelle le «nous œcuménique », à savoir notre plus grand dénominateur commun. Quand les planétologues posent la question de savoir si nous sommes seuls dans l'univers, ce «nous » ne renvoie pas nécessairement au concept biologique de vie. Pour l'astrobiologie contemporaine, le «nous p peut parfois faire référence aux êtres dont la constitution est proche de celle des roches, des océans, ou même des planètes. 


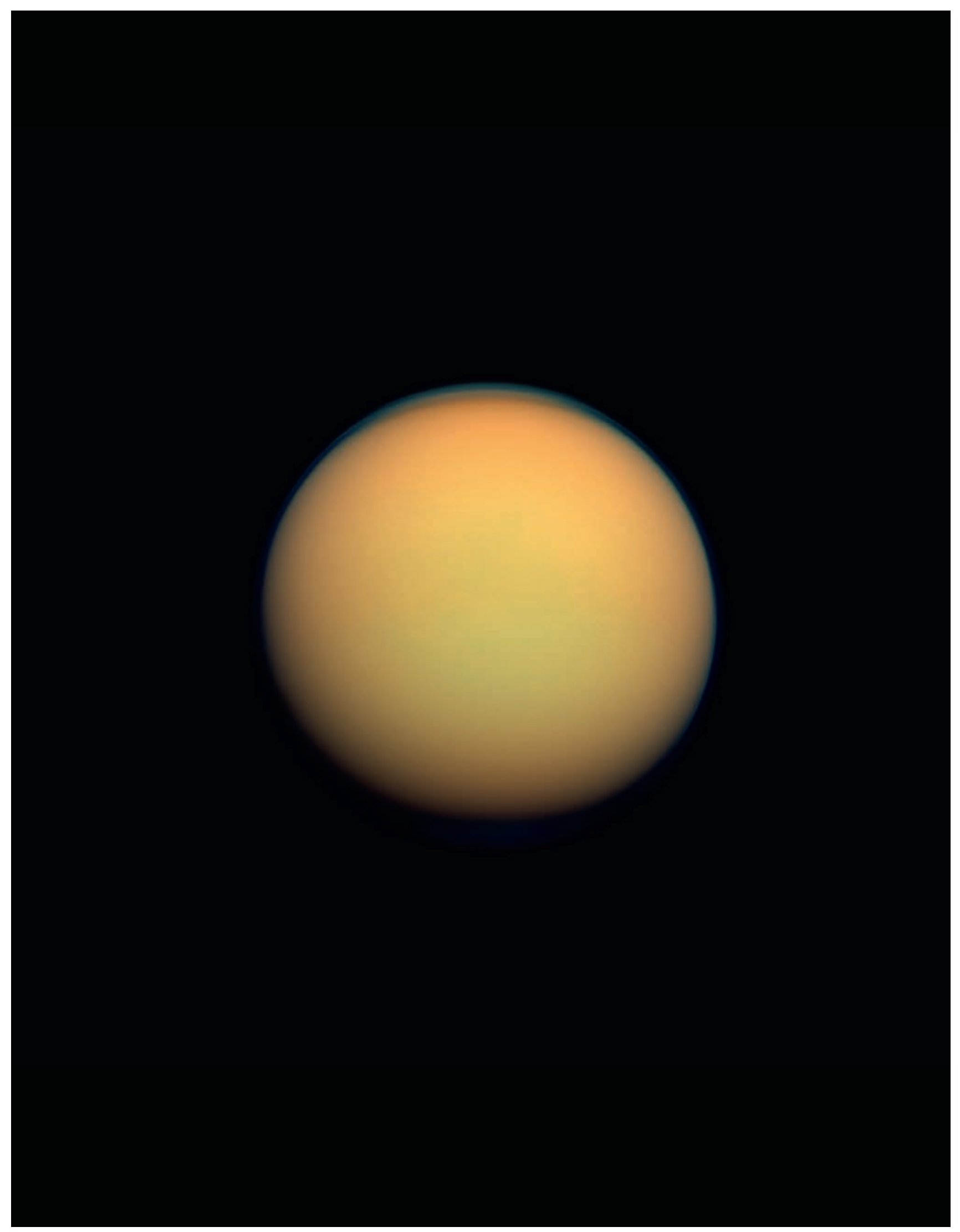


«... deux planètes opposées par des conditions si différentes que les premiers témoins ne purent croire qu'elles fussent également humaines. »

Claude Lévi-Strauss (Tristes Tropiques, 1955) à propos de la découverte du Nouveau Monde.

\section{Introduction}

En Occident, le concept biologique de vie joue le rôle d'un «nous» œcuménique: au niveau le plus inclusif, nous faisons partie des «êtres vivants», et cette condition d'être «vivant» est la caractéristique la plus générale du «nous». Ce «nous» œcuménique semble aller de soi. Ainsi, on sait tous intuitivement que nous, êtres humains, avons quelque chose en commun avec les autres organismes vivant dans la biosphère terrestre. Et on sait aussi, toujours intuitivement, que les êtres humains sont fondamentalement distincts des molécules, des volcans ou des lunes, par exemple. Mais ce consensus est-il si général qu'il y paraît à première vue? En examinant de près l'astrobiologie contemporaine et la recherche spatiale, en particulier l'exploration de la plus grande lune de Saturne, Titan, je voudrais montrer que ce n'est en réalité pas le cas. II est bien entendu que les pronoms, parmi lesquels le «nous», prennent des sens extrêmement variés. D'un point de vue technique, ce sont ce qu'on appelle des «déictiques», c'est-à-dire que leur signification dépend du contexte dans lequel on les utilise. Les anthropologues ont récemment beaucoup écrit sur la contingence contextuelle des pronoms (par exemple Viveiros de Castro 1998). Mais presque rien n'a été dit sur la délimitation de ce qu'ils désignent. Selon moi, le «nous» œcuménique, tout au moins en Occident, a eu tendance à être remarquablement stable sur de longues périodes de l'histoire et est globalement indépendant de tout contexte national. Au cours des deux derniers siècles, il a en effet relativement peu évolué. La notion biologique de vie, si l'on met de côté les querelles ponctuelles autour de sa délimitation exacte (par exemple «les virus sont-ils vivants?»), est transnationale: elle est aussi solidement ancrée dans la culture française que dans la culture britannique ou japonaise, par exemple. Si les universitaires d'aujourd'hui ont peut-être une compréhension plus subtile de la vie que les contemporains de Charles Darwin, le concept n'a pas fondamentalement changé depuis la fin du $x x^{e}$ siècle. Les scientifiques ont certes détecté la présence de micro-organismes dans des endroits aussi improbables que des sources chaudes ou des lacs d'asphalte, mais ces découvertes n'ont pas radicalement bouleversé l'acception commune la plus englobante du «nous»; elles n'ont fait que la compléter.

Cependant, cette stabilité sur le long terme ne signifie pas que des transformations conceptuelles importantes et soudaines sont à exclure. Ainsi, l'anthropologue Stefan Helmreich soutient l'idée que le concept de vie est en train de subir de profondes mutations: «ll est aujourd'hui en cours de transformation, voire de dissolution. » (Helmreich 2011: 671) De la même façon, sa collègue Gísli Pálsson (Ingold et Pálsson 2013: 229) souligne que la vie en tant que phénomène biosocial s'émancipe des divisions classiques entre disciplines académiques. Helmreich a montré que l'astrobiologie

\section{ci-contre}

fig. 1

Titan, la plus grande lune de Saturne, ressemble à une balle orange et floue depuis la sonde spatiale Cassini-Huygens, 2009 (C) NASA/JPL-Caltech/ Space Science Institute. 


\section{ci-contre}

fig. 2

Scène du film 2001

l'Odyssée de l'espace, de Stanley Kubrick, 1968

Photo Archive Photos / Metro-Goldwyn-Mayer/ Getty Images, (C) Warner Bros. est l'un des domaines où ce changement est particulièrement flagrant. Je pense qu'il a raison, mais j'ajouterai que ce genre de transformation n'est pas propre à notre époque. Une perspective historique plus large permet au contraire de constater que les conceptions du «nous» œcuménique même si ces phénomènes de longue durée sont remarquablement solides et stables - ont subi des transformations relativement brutales à des moments spécifiques de l'histoire. C'est pourquoi ces phénomènes sont particulièrement bien adaptés au type d'analyse structurale proposée par des anthropologues tels que Philippe Descola (2005). Mais mon objectif se veut ici simplement exploratoire. Je me limiterai à une ébauche rapide et provisoire de l'histoire du "nous» dans son sens le plus englobant, tel qu'il s'est développé au cours du dernier millénaire en Europe. Je montrerai ensuite les liens que l'on peut tisser avec les développements actuels dans le champ de l'astrobiologie, qui apparaît comme un foyer d'innovations conceptuelles très audacieuses, en tout cas en ce qui concerne le "nous" œcuménique et le sens de la «vie». Mais avant de nous lancer, faisons un détour par la Suède.

II semble évidemment y avoir de grandes différences entre Saturne et la Terre d'un côté, et vous et moi de l'autre. Ce sont des planètes, alors que nous sommes des êtres humains. Mais l'évidence de ces catégories dépend entièrement de la perspective adoptée. En termes de taille et de forme, la distinction va bien entendu de soi. Mais si l'on prend un autre critère, alors ce n'est peut-être plus si évident. Les hommes et leur planète font leur révolution annuelle autour du Soleil à une vitesse moyenne de $30 \mathrm{~km} / \mathrm{s}$. Saturne, en revanche, met trente ans, à une vitesse orbitale de $10 \mathrm{~km} / \mathrm{s}$. De ce point de vue, la différence première est entre les objets à révolution rapide les hommes et la Terre - et les plus lents - Saturne. Prenons encore un autre critère, par exemple la densité. Alors la Terre devient l'aberration, tandis que les hommes et Saturne sont plutôt semblables. Le contraste s'établit ici entre des corps dont la densité est relativement élevée, comme la Terre, et des corps de densité moindre les hommes et Saturne. Ce qu'on finit par qualifier de «nous » dépend ainsi toujours des critères de jugement appliqués.

En 2012, au cours d'une réception à l'hôtel de ville de Stockholm, de jeunes participants à un colloque d'astrobiologie débattaient de manière vive du caractère arbitraire des étiquettes astronomiques. La discussion avait été déclenchée par la relégation de Pluton du statut de "planète" à celui de "planète naine", que certains trouvaient encore difficile à accepter. Quelqu'un avança l'idée que les «lunes géantes" comme Ganymède (Jupiter) ou Titan (Saturne) méritaient elles aussi le statut de «planète». Quelqu'un d'autre souligna que la frontière était non seulement floue entre lunes et planètes, mais aussi entre planètes et étoiles. Par convention, Jupiter et Saturne sont ce qu'on appelle des "géantes gazeuses", mais on pourrait également les considérer comme des «étoiles ratées " puisqu'elles possèdent différentes caractéristiques communes aux naines brunes et aux objets stellaires plus légers. 


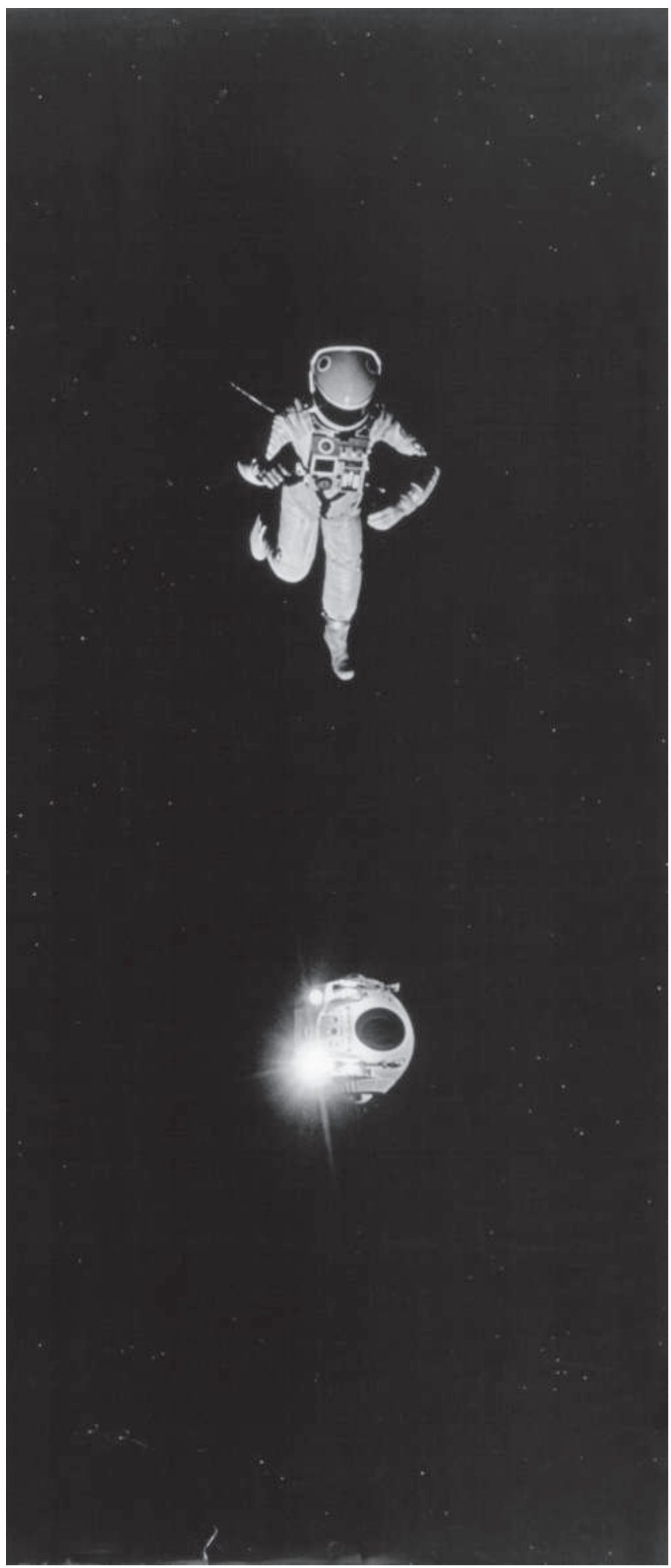


Après une journée intense de colloque, l'atmosphère de la soirée était détendue, quoique gentiment animée. D'un côté se trouvaient les partisans des labels historiques traditionnels. «Si on commence à jouer avec des distinctions établies depuis longtemps et communément admises telles que la différence entre planètes et étoiles, on aboutira à une situation peu souhaitable où tout sera permis. » En face, certains prenaient un malin plaisir à contredire leurs collègues: «Communément admises par qui?, demanda le jeune doctorant hétérodoxe cité plus haut. II est possible que des extraterrestres observant le système solaire préfèrent prendre comme critère la densité moyenne, auquel cas les hommes et Saturne seraient semblables, alors que la planète Terre appartiendrait à une tout autre espèce. Nos catégories conventionnelles ne sont peut-être pas si évidentes que nous avons tendance à l'imaginer. »

Cette petite scène illustre un aspect central de l'astrobiologie, cette science qui se donne pour objectif de déceler les traces d'une vie passée et/ou présente dans l'espace: son empressement à saisir, voire à repenser radicalement «notre» place dans l'univers. Toutes les communications présentées lors de ce colloque exploraient d'une façon ou d'une autre cet aspect des choses: biochimies hypothétiques, extrêmophiles, astéroïdes primitifs, photosynthèse exotique en système stellaire binaire, etc. L'astrobiologie, qui couvre un champ de sujets étonnamment vaste, fait intervenir toutes les sciences naturelles et repose à la fois sur des observations empiriques et des spéculations théoriques. Cela va de l'étude de l'histoire géologique de Mars (qui, il y a bien longtemps, était recouverte de fleuves et de mers) à la détermination de la couleur de la végétation (violette ou noire, et non plus verte) sur les exoplanètes qui ressemblent à la Terre. Le but affirmé de l'astrobiologie est, de manière générale, d'étudier la présence de vie dans l'univers tout entier. Si les origines de la vie terrestre constituent certes l'un de ses thèmes de recherche les plus importants (les scientifiques de la NASA ont cru trouver des fossiles microscopiques de bactéries martiennes dans une météorite découverte dans les Allan Hills, en Antarctique), l'astrobiologie est devenue un champ de recherche en pleine expansion disposant de ses propres revues spécialisées, de collections éditoriales, de colloques et de sociétés savantes. Aujourd'hui, des chercheurs venant d'horizons disciplinaires très variés se tournent vers elle: physiciens, biochimistes, géologues, planétologues, biologistes, astronomes et philosophes des sciences, entre autres. Dans cet article, je souhaiterais examiner la façon dont les astrobiologistes ne cessent de remettre en question et de redéfinir le grand fossé qui sépare le terrestre de l'extra-terrestre, cette entreprise faisant à plus d'un titre écho au problème anthropologique du «nous» et du «eux» auquel il est fait référence dans la citation en exergue de ce texte. Dans ce qui suit, je voudrais démontrer que les conclusions des anthropologues, dont Claude Lévi-Strauss, à propos de la rencontre entre les premiers explorateurs européens et les Indiens d'Amérique peuvent également être appliquées, dans une certaine mesure au moins, à la prétendue division entre Terriens et extraterrestres. Ma démonstration repose sur le cas particulier de la plus grande lune de Saturne, Titan, grande favorite de l'astrobiologie en raison de sa très forte analogie avec notre planète au sein du système solaire. Instruments téléguidés et vaisseaux spatiaux robotisés, telle la sonde Cassini-Huygens, ont remplacé les expéditions maritimes et 
les explorateurs en chair et en os. Mais, contrairement à ce qu'on pourrait penser, on retrouve en réalité les mêmes problèmes conceptuels fondamentaux (voir Battaglia 2005; Pálsson 2009). La présente étude repose sur un travail de terrain mené dans différents endroits depuis 2009 et sur une analyse de sources électroniques et de textes très variés. J'ai principalement effectué ma recherche en Europe (dans les locaux de l'Observatoire européen austral à Garching, près de Munich, et lors de différents colloques, rencontres et ateliers d'astrobiologie organisés au Royaume-Uni, en Écosse, en France, au Danemark et en Suède). J'ai également interrogé des astrobiologistes du Jet Propulsion Laboratory à Pasadena, en Californie. Cette étude s'inscrit dans un projet de recherche plus vaste visant à étudier l'exploration spatiale actuelle d'un point de vue anthropologique.

\section{La plus grande lune de Saturne, une exploration qui se poursuit}

En août 1907, l'astronome catalan Josep Comas i Solà observait Titan à l'aide de son modeste télescope de $38 \mathrm{~cm}$ installé dans l'observatoire Fabra de Barcelone. II remarqua que les contours du disque lunaire étaient moins brillants que son centre, un phénomène connu sous l'expression technique d'«assombrissement centre-bord». C'était là une découverte passionnante qui prouvait que Titan possédait une atmosphère. À l'époque, l'observation de Comas i Solà ne fit pas l'unanimité, et il fallut attendre le début des années 1940 pour qu'elle fût validée par son collègue américanohollandais, Gerard Kuiper, de l'observatoire McDonald au Texas. À l'aide de la spectroscopie, une technique permettant d'établir l'«empreinte»caractéristique d'un rayon lumineux, Kuiper parvint à identifier du méthane, apportant ainsi la preuve définitive de l'existence d'une atmosphère autour de Titan. Par conséquent, le plus grand satellite de Saturne occupe une place très particulière au sein de notre système solaire: aucune autre lune d'aucune autre planète ne possède une atmosphère aussi dense (Gilmour et Sephton 2004: 172). Grâce à la présence inédite d'une atmosphère, Titan est, à ce jour, le corps céleste ressemblant le plus à la Terre. II n'est donc pas surprenant que cette lune géante (elle est un peu plus grande que Mercure et de loin la plus grosse lune de Saturne) soit devenue l'une des cibles privilégiées des missions d'exploration spatiales modernes. En novembre 1980, la sonde Voyager I passa à moins de 5000 km de Titan. On espérait pouvoir obtenir un premier aperçu de la surface de cette lune, mais il s'avéra qu'elle était entièrement enveloppée d'un voile orangé, dense et informe. Voyager I n'était malheureusement pas équipée de caméras infrarouges ni d'instruments capables de voir au travers de ce voile opaque.

Quand la sonde Cassini-Huygens a été lancée depuis Cape Canaveral en octobre 1997, le mystère Titan fascinait toujours autant. Les planétologues savaient désormais que, tout comme celle de la Terre, l'atmosphère de Titan était principalement composée d'azote moléculaire (à plus de $90 \%$ de la surface; le méthane détecté par Kuiper ne représente pas plus de $5 \%$ ). Mais, fait plus passionnant encore, ils avaient découvert la présence d'une diversité considérable d'hydrocarbures, comme de l'éthane ou du propane, qui interagissent de manière complexe, laissant penser que cette atmosphère n'est pas en équilibre chimique. Les scientifiques se sont alors rendu compte qu'ils avaient là un véritable «laboratoire naturel» 


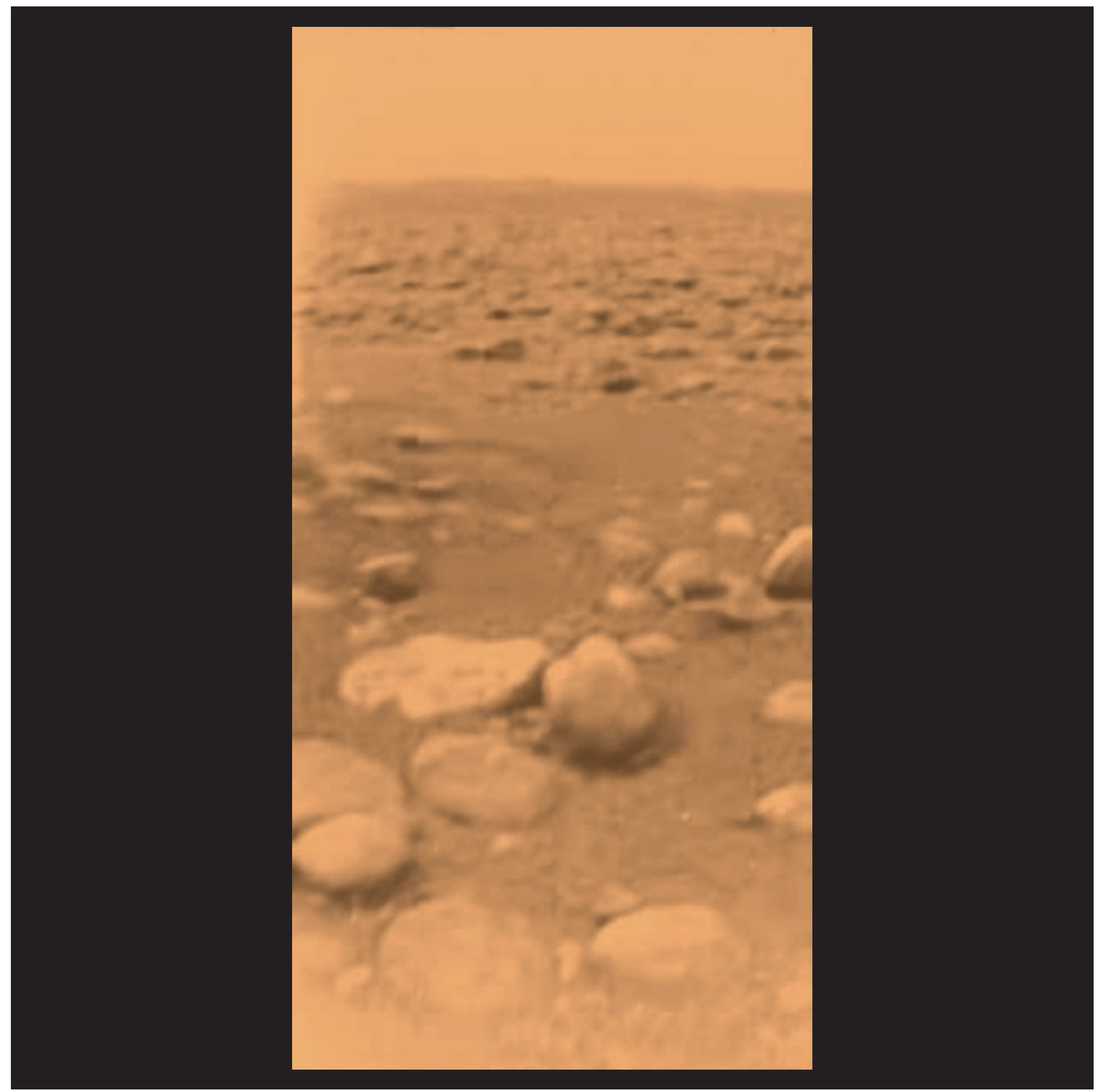


leur offrant l'occasion inédite d'étudier la chimie organique et son évolution sur le long terme. On comprend mieux, dès lors, les espoirs fondés sur la sonde Cassini-Huygens quand celle-ci a atteint le système de Saturne (qui comprend plus de soixante lunes et satellites mineurs) en juillet 2004 après un voyage de sept ans. Cette sonde robotisée est le fruit d'une collaboration entre l'agence spatiale américaine, la NASA, son équivalent européen, I'ESA, et l'agence spatiale italienne, l'ASI. À bord du vaisseau, qui mesure sept mètres de haut sur quatre de large, des ordinateurs exécutent les instructions envoyées par la NASA depuis le Jet Propulsion Laboratory en Californie et commandent les différents instruments techniques dont il est équipé. À la fin de l'année, la sonde s'est scindée en deux: le module Huygens a été largué et parachuté dans l'atmosphère de Titan, avant d'atteindre sa surface en janvier 2005. C'était la première fois qu'on tentait de faire atterrir un module spatial sur un corps céleste du système solaire externe.

La descente de deux heures et demie s'est déroulée sans incident et Huygens a transmis des informations pendant trois heures après son atterrissage. Cassini a relayé les précieuses données collectées jusqu'à la Terre, et continue à ce jour d'enregistrer et de cartographier le climat et la surface de Titan (Lorenz et Mitton 2008; Coustenis et Taylor 2008). L'un des résultats les plus intrigants de la mission est que la température et la pression moyennes de Titan sont étonnamment proches du point triple du méthane $(90,7 \mathrm{~K}$ pour 1,6 bar). Cela signifie que le méthane peut se présenter sous forme gazeuse, liquide et solide, tout comme l'eau sur Terre (où la température et la pression moyennes coïncident presque exactement avec le point triple de l'eau). La surface de Titan est en grande partie composée d'eau, mais étant donné les conditions de froid extrême qu'on y observe, celle-ci n'existe que sous la forme d'une glace aussi dure que la pierre sur Terre. Ainsi, les rôles changent: le méthane joue celui de l'eau, tandis que l'eau devient le substitut de la pierre. On estime que cette lune possède une croûte de glace d'environ $120 \mathrm{~km}$ d'épaisseur recouvrant probablement un océan d'eau liquide contenant de l'ammoniaque, qui agit comme un antigel (Fortes et al. 2000; Lorenz et al. 2008; Coustenis et Blanc 2012). Contrairement à ce que bien des planétologues s'attendaient à trouver au départ, les températures très basses observées sur Titan n'ont manifestement pas créé un monde congelé.

Sur Titan, il y a des nuages, de la pluie et du vent, et même ce qui semble être un ouragan permanent au pôle sud. Mais, tandis que sur Terre, le climat repose sur des échanges d'eau, sur Titan, il s'agit de méthane: Cassini a permis d'observer que, pour des raisons qu'on ne comprend pas encore très bien, les nuages de méthane condensé semblent se limiter au tourbillon de turbulences du pôle sud et à une bande étroite située dans l'hémisphère sud. Les observations réalisées par le télescope spatial Hubble avaient déjà montré que le voile opaque orangé change régulièrement de forme: parfois c'est le pôle nord de Titan qui est le plus brillant (c'est-à-dire qu'il est couvert par une brume plus dense), parfois c'est le pôle sud. Cassini a contribué à expliquer ce phénomène en confirmant l'existence d'un régime de vents changeants qui déplacent le voile nuageux de façon régulière d'un pôle à l'autre. De manière générale, la répartition de la pression et de la température dans l'atmosphère de Titan ressemble de

\section{ci-contre}

fig. 3

Première vue en couleur de la surface de Titan, 14 janvier 2005 (c) NASA/JPL/ ESA/University of Arizona. 
façon troublante à celle qu'on trouve sur Terre, même si son amplitude est plus importante verticalement (du fait, entre autres, de la plus faible gravité sur cette lune) et que les températures atteignent des valeurs beaucoup plus basses. La présence d'une atmosphère influence considérablement les propriétés de la surface d'un corps céleste puisque le climat peut déposer des matériaux ou les éroder. La mission Cassini-Huygens a en effet confirmé qu'on trouve sur Titan des paysages très diversifiés, que Ralph Lorenz et Jacqueline Mitton (2008: 19) décrivent ainsi:

Les vues les plus détaillées des paysages de Titan nous sont parvenues grâce aux images enregistrées par Huygens lors de sa descente et par le radar de Cassini qui, à chaque passage, réalise à travers la surface une coupe de quelques centaines de kilomètres de large sur plusieurs milliers de kilomètres de long. Dès le départ, ces balayages ont révélé une diversité de paysages aussi vaste que celle qu'on trouve sur Terre: des dunes formées par le vent, des cratères, des chaînes de montagnes, et même quelque chose qui ressemble à un dôme volcanique. Le plus intrigant, ce sont ces éléments qui ressemblent à des canaux, des rivages et des traces de lacs, indiquant qu'il y a eu par le passé du liquide sur Titan, ou bien que ce liquide s'écoule de temps en temps sans former d'océans ni de grands lacs. Huygens s'est posé tout près de ce qui ressemble à une rive entre une zone claire et une zone plus foncée et a transmis des images d'embranchements de canaux de couleur foncée sillonnant la zone la plus claire.

L'interprétation de ces différents éléments reste très hypothétique. Si les zones sombres étaient réellement des mers ou des lacs d'hydrocarbures liquides, Cassini aurait dû enregistrer des phénomènes de réflexion optique (tel l'éclat du soleil sur une surface polie). Dès lors, il n'est pas certain que les formes observées correspondent bien à des lignes côtières et à des îles. L'un des facteurs explicatifs est peut-être que, sur Titan, où la gravité équivaut à $15 \%$ de celle de la Terre, le vent forme sur les étendues liquides des vagues beaucoup plus hautes et beaucoup plus larges que celles qu'on trouve sur les océans de notre planète. On a calculé, par exemple, qu'avec un vent de 3 sur l'échelle de Beaufort (c'est-à-dire une brise légère), la vague moyenne sur Terre mesure $0,6 \mathrm{~m}$ de haut sur $11 \mathrm{~m}$ de large, tandis que sur Titan elle mesurerait 4,5 m de haut sur $105 \mathrm{~m}$ de large (Gilmour et Sephton 2004: 192). L'océanographie d'une mer de méthane/ éthane n'a donc pas grand-chose à voir avec les mers d'eau qui nous sont familières à nous, Terriens. Et même si, sur son site d'atterrissage, la sonde Huygens a trouvé des galets qui semblaient avoir été polis par un élément liquide quelconque, rien ne permet d'affirmer de façon définitive qu'il y avait auparavant sur Titan des fleuves, des lacs ou des mers.

Dans le même temps, en revanche, certains faits ont été établis avec plus de certitude. Au cours d'un survol effectué plus tard la même année, Cassini a repéré une étrange tache sombre en forme de croissant près du pôle sud de Titan. Celle-ci a été baptisée «Ontario Lacus» en raison de sa ressemblance approximative avec l'un des Grands Lacs nord-américains. En réalité, il pourrait tout aussi bien s'agir d'une vaste étendue de suie ou 
de toute autre substance non liquide. Lors de son dix-septième survol de Titan, Cassini a fini par détecter à proximité du pôle nord une série de lacs de tailles et de formes très différentes (Stofan et al. 2007). II s'agirait de corps de méthane et/ou d'éthane liquide. En outre, on a trouvé des indices incontestables de l'évolution du niveau du liquide: des sédiments se sont déposés sur le lit de ces lacs en y laissant des traces distinctives parallèles à la rive, un peu comme celles qui se déposent sur les parois des baignoires. Des traces circulaires du même type ont également été découvertes autour de l'Ontario Lacus, ce qui suggère la présence de gigantesques étendues liquides à la surface des deux régions polaires de Titan (Barnes et al. 2009). À ce jour, on a identifié 655 de ces corps liquides, dont la taille varie de $10 \mathrm{~km}^{2}$ à plus de 100000 km² (Coustenis et Blanc 2012: 186).

\section{Des espérances déçues}

Ces récentes découvertes sont tout à fait passionnantes, pour les planétologues comme pour le grand public, et on comprend dès lors pourquoi on dit que Titan est «un monde d'une complexité aussi grande que celle de la Terre». Mais les spécialistes qui adoptent une perspective à plus long terme font parfois part d'une certaine frustration. Selon un jeune planétologue: «Si Huygens pouvait revenir parmi nous, il serait extrêmement déçu. " Un de ses collègues plus âgés ajouta: «Nous devons admettre, à notre plus grand regret, que le système solaire est bien plus stérile que nos prédécesseurs ne l'imaginaient. » Puisqu'il n'est pas rare d'entendre ce genre de propos, il faut peut-être dire un mot sur Christian Huygens, le physicien et astronome qui, au XVII ${ }^{e}$ siècle, découvrit Titan et donna son nom à la sonde qui s'y posa pour la première fois. Figure intellectuelle néerlandaise majeure, il fut un pionnier dans l'étude des «petits gentilshommes tournant autour de Jupiter et Saturne », ainsi qu'il appelait affectueusement les satellites de ces planètes géantes. Grand empiriste, c'est aussi lui qui découvrit les anneaux de Saturne et contribua à élucider la nature de la nébuleuse d'Orion grâce à un télescope conçu par ses soins. Mais il fut également un grand spéculateur, comme en témoigne l'extrait suivant d'une lettre adressée à son frère Constantijn:

Un homme qui partagerait l'opinion de Copernic, selon laquelle notre Terre est une planète, ronde et éclairée par le Soleil, comme les autres [Huygens était un fervent partisan d'un modèle héliocentrique du système solaire, idée encore minoritaire à son époque], ne peut s'empêcher de temps à autre d'imaginer qu'il n'est pas improbable que les autres planètes aient elles aussi leurs parures et leur mobilier, plus encore leurs habitants comme cette Terre qui est la nôtre: en particulier s'il considère les dernières découvertes qui ont été faites depuis Copernic des compagnons de Jupiter et de Saturne, des plaines et des pays vallonnés de la Lune, autant d'arguments en faveur d'une relation et d'une affinité avec la Terre, ainsi qu'une preuve de la vérité de ce système. Nous évoquions souvent cela, je m'en souviens, mon bon frère, penchés sur un large télescope pour regarder ces corps... (Huygens 1698, tome I)

Ce sont là les premières lignes du Cosmotheoros, que l'on considère aujourd'hui comme l'un des textes fondateurs de l'astrobiologie et double page suivante

fig. 4

Canaux au milieu des dunes de la région de Xanadu sur Titan, vus par les radars de la mission Cassini-Huygens. Photo NASA/JPL-Caltech/ASI. 


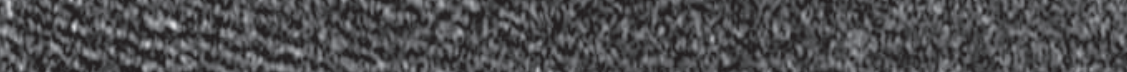
20.

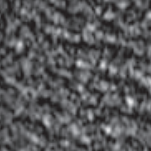

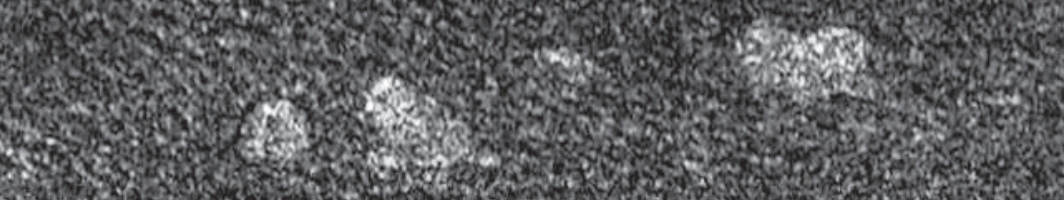

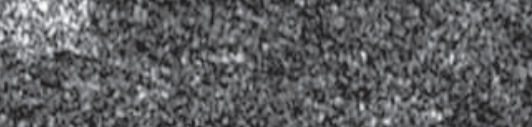

Yas $x^{4}$

is.

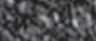

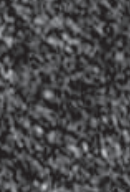

3.0.

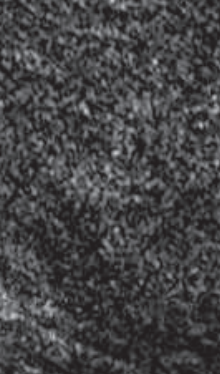

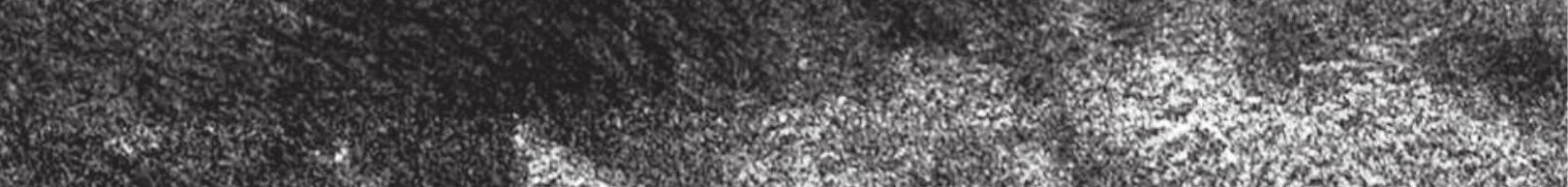

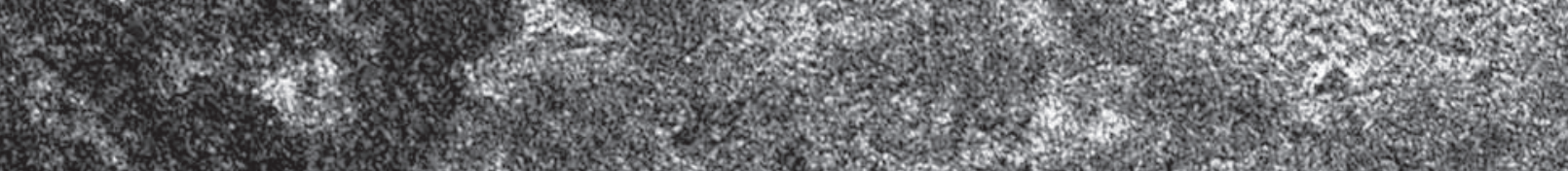
Whe 3. Sin 32 (5) iston 

153.

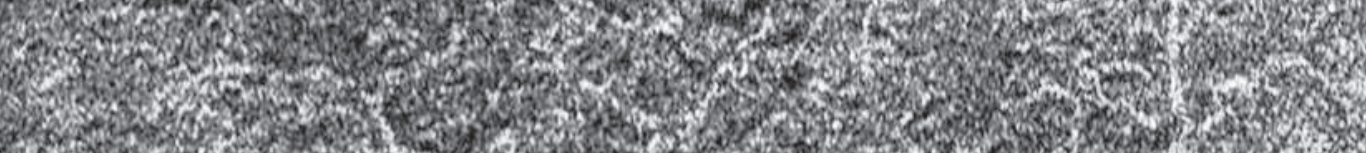

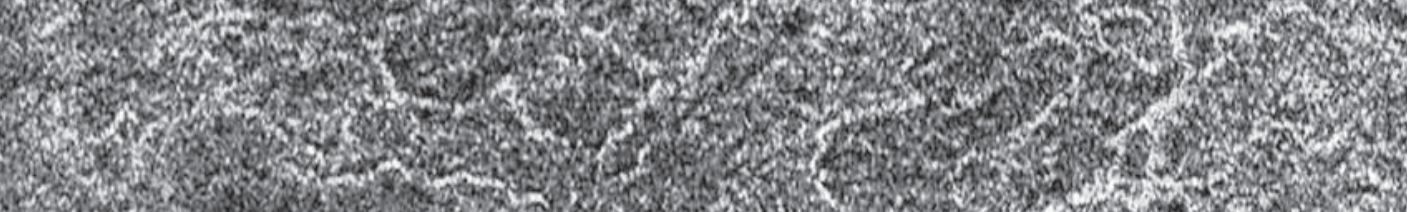
.

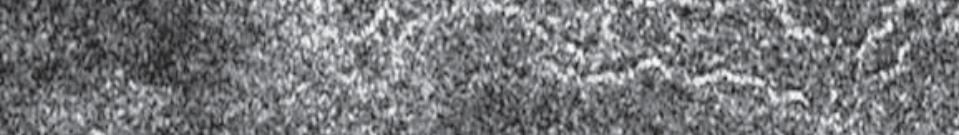
1. 1. 2.

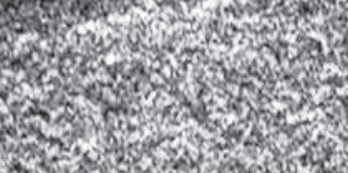
(6.

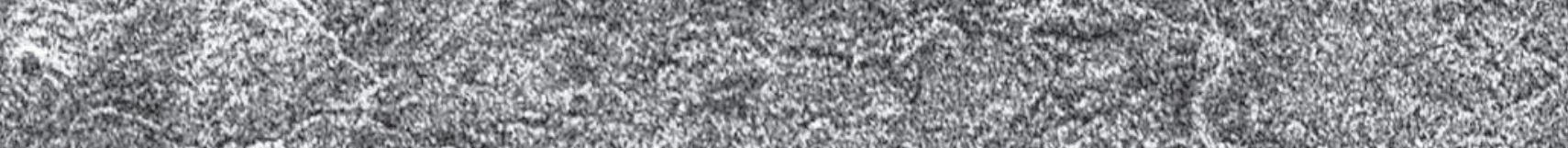

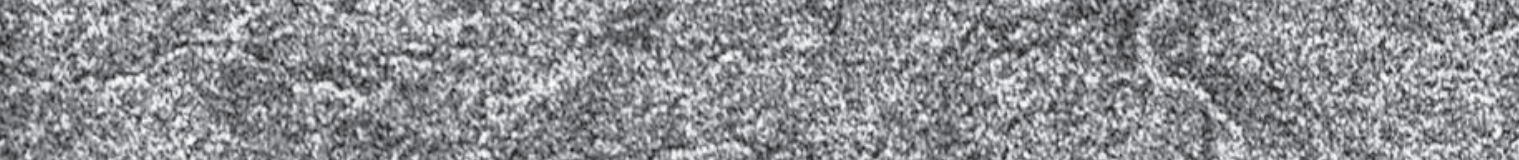

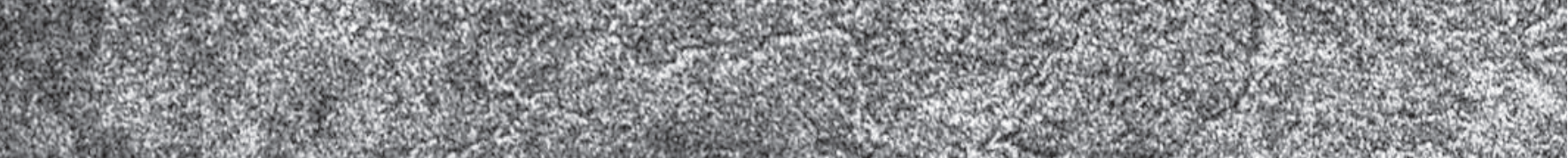

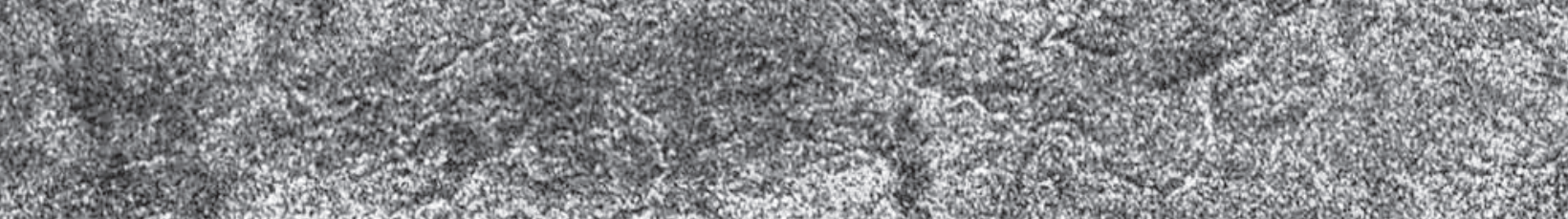

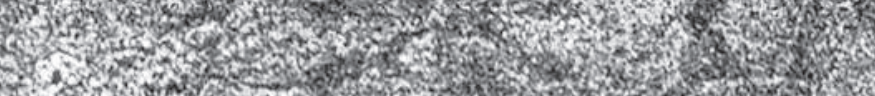
1.

$x+2 x^{2}$
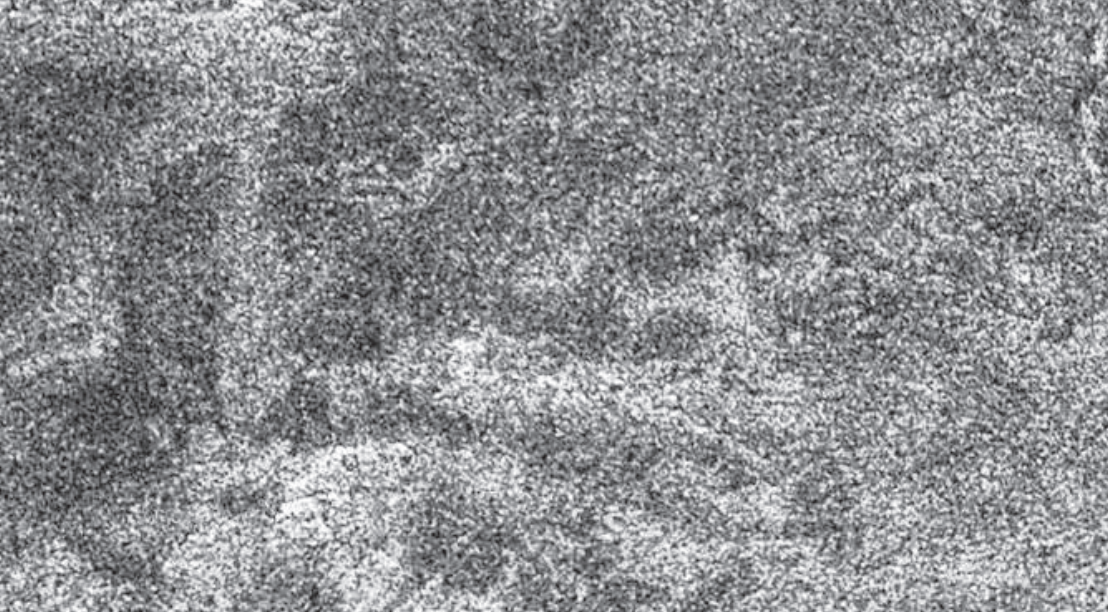

$$
\begin{array}{cc}
3 \\
3
\end{array}
$$

Hoin

i.

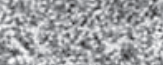

(2) (x)

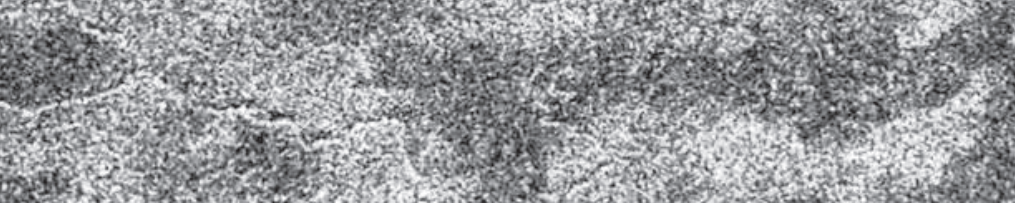

Desis

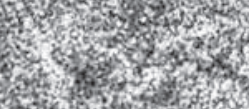

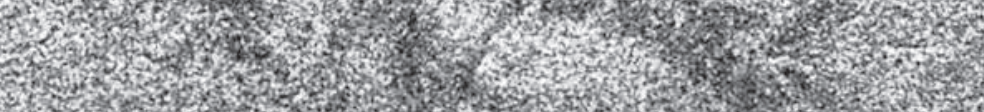


dans lequel Huygens avance la thèse selon laquelle tous les corps célestes sont égaux «en beauté et en dignité ». Il affirme qu'il existe une multitude de mondes «tout aussi habités et ornés que le nôtre». Il était convaincu que les planètes et les lunes du système solaire étaient peuplées par toute une variété d'animaux et de plantes, bien que sans doute «très peu différents de ceux que nous pouvons voir et observer ici». Partant de l'idée selon laquelle ce «mobilier» devait avoir été placé là pour être utilisé et contemplé, il suggéra l'existence d'extraterrestres ou, pour reprendre ses termes, de «planéteriens": "Peut-être pas des hommes comme nous, mais des créatures d'un genre ou d'un autre, douées de raison. » (Ibid.) Sa prédiction selon laquelle le télescope aurait un jour levé le voile sur un monde d'une complexité aussi grande que celui que permit de découvrir une autre invention décisive $d u x v \|^{e}$ siècle, le microscope, ne fut jamais réalisée. En effet, on ne peut pas vraiment dire que les corps célestes de notre système solaire grouillent de ces formes baroques de vie extraterrestre imaginées par Huygens. En dépit des avancées majeures de l'astrobiologie ces dernières décennies, les scientifiques s'accordent tous aujourd'hui à dire que rien ne prouve l'existence d'une vie extraterrestre (voir Dick 1998). Les découvertes comme celles de la sonde Cassini-Huygens, pour aussi stimulantes qu'elles soient, n'ont apparemment pas permis de démontrer que la vie terrestre n'était pas un phénomène unique.

L'astrobiologie telle qu'elle se pratique aujourd'hui se présente volontiers comme éminemment interdisciplinaire. Elle couvre tous les grands domaines scientifiques: la physique, la chimie, la biologie, la géologie et l'astronomie. Ses spécialistes viennent d'horizons très divers: ils sont astrophysiciens, biochimistes, microbiologistes, planétologues, géochimistes, étudient les microfossiles, la génomique comparative, etc. L'astrobiologie travaille donc sur des échelles qui vont du moléculaire au galactique, de l'infiniment petit à l'infiniment grand. Dans ce domaine, les chercheurs semblent avoir conscience du champ démesurément vaste abordé par leur jeune discipline. Lors du colloque d'astrobiologie auquel j'assistai récemment en Suède, plusieurs participants évoquèrent l'image d'un retour à une science totale telle qu'elle se pratiquait à la Renaissance. "L'astrobiologiste idéal, me confia un participant lors d'une pause, est une sorte de Léonard de Vinci : il doit posséder un savoir encyclopédique. " Dans ce qui va suivre, j'aborde l'astrobiologie dans une perspective anthropologique. Je prends comme point de départ la question au cœur de toutes les études astrobiologiques: “Sommes-nous seuls dans l'univers?» Plus spécifiquement, je m'interroge sur le point le moins étudié sans doute de ladite question, à savoir le «nous». À première vue, il paraît évident que, quand ils disent «nous», les astrobiologistes font tout simplement référence à «la vie sur Terre». Tout ce qui peut être qualifié d'organisme vivant peut être rangé dans le «nous». Cependant, la définition de la «vie» pose de grandes difficultés, qui dépendent en partie de la perspective disciplinaire adoptée (voir Morange 2009). Les spécialistes de biologie moléculaire ont tendance à prendre en compte des critères différents de ceux invoqués par les spécialistes de l'écologie ou de l'évolution, par exemple. Les non-biologistes préfèrent même parfois des définitions assez excentriques; je pense notamment au chimiste et planétologue James Lovelock (2000) qui, selon sa célèbre hypothèse Gaïa, considère la Terre comme une entité vivante. 
Les astrobiologistes, qui estiment que leur tâche principale consiste à identifier clairement toute vie extraterrestre, sont très conscients de cette difficulté. Le fait que personne n'ait réussi à proposer une définition absolue est souvent perçu comme un échec. Pour reprendre l'expression d'un astrobiologiste rencontré lors du colloque en Suède, c'est le signe qu'«on rate quelque chose». II insista par ailleurs sur le fait que la «nature insaisissable» du concept de vie devrait faire l'objet de recherches plus rigoureuses. Et c'est peut-être là que l'anthropologie peut s'avérer utile.

\section{Brève histoire du "nous" œcuménique moderne}

D'un point de vue anthropologique, la notion de vie tient lieu de «nous» œcuménique dans nos sociétés occidentales modernes. C'est en effet le «nous» le plus inclusif auquel on peut penser. II comprend tous les organismes qui figurent sur ce qu'on appelle l'«arbre de la vie», une représentation dont l'influence fut sans précédent et qui permit à Darwin de figurer l'idée que tous les êtres vivants sont reliés entre eux et partagent une ascendance commune. Voilà pourquoi on n'est pas choqué d'entendre parler de «nous, hommes et animaux », ou même «nous, hommes et microbes ». Tout comme l'anthropologie, qui étudie principalement «nous, hommes », la biologie a également pour objet d'étude un «nous» ("êtres vivants»): elle considère même que les formes de vie les plus étranges, tels ces extrêmophiles vivant dans les fissures volcaniques des fonds marins, sont elles aussi des êtres vivants, c'est-à-dire «comme nous», des êtres familiers mais pas extraterrestres (par défaut). L'anthropologie comme la biologie mettent toujours l'accent sur ce qui «nous» est commun. C'est ce qui rend ces deux sciences un peu particulières; les autres sciences modernes ont plutôt tendance à minimiser, voire à nier complètement une quelconque ressemblance entre les êtres vivants et leur objet de recherche. De toute façon, il serait bien étrange de parler de «nous, hommes et photons», "nous, êtres vivants et molécules», «nous, hommes et océans» ou encore «nous, êtres vivants et planètes ». Si l'on peut définir l'anthropologie et la biologie comme des «sciences de la première personne» (même si, j'en conviens, la formule est un peu maladroite), il semblerait que la physique, la chimie, les sciences de la Terre et l'astronomie soient, pour une raison ou une autre, toujours perçues comme des «sciences de la troisième personne». Quoi qu'il arrive, un quark, un polymère, un volcan et une galaxie ne sont à nos yeux «pas comme nous ", c'est-à-dire qu'ils sont extra-terrestres (alien).

La division entre les sciences dont l'objet premier nous est a priori familier et celles dont l'objet nous semble a priori étrange reflète-t-elle une vérité profonde ou n'est-elle en réalité qu'une caractéristique contingente de la cosmologie moderne? La question reste ouverte et mérite une étude ethnographique plus approfondie. Mais le point fondamental est ici que la vie terrestre joue le rôle de «nous» œcuménique dominant. On oublie souvent que cette conception particulière est le produit d'une histoire spécifique. En Europe occidentale, elle eut différents précurseurs sur lesquels il faut peut-être s'arrêter un moment. Je pense en particulier aux Grecs et à l'idée de monde connu ou habité, l'oikouménè, aux notions liées à la chrétienté telles qu'elles se sont développées au Moyen Âge et au concept d'espèce humaine apparu à la Renaissance, qui s'est développé et cristallisé pendant les lumières. 
Pour les cartographes occidentaux du Moyen Âge, le monde connu comprenait l'Europe, l'Asie et l'Afrique, chaque continent étant entouré par un océan. Ils avaient hérité cette image du monde, centrée sur le Bassin méditerranéen, de la géographie classique grecque et latine. Ces mappae mundi ne proposèrent pas de représentation plus évoluée du monde; la relation entre le «nous » du connu et le «ils » de l'inconnu, entre œkumène et anti-œkumène, resta un grand sujet de débat philosophique. Très tôt, des auteurs comme saint Augustin et Isidore de Séville s'interrogèrent sur les Antipodéens, ceux qui habitaient au-delà de l'œkumène. Comme l'a montré Alfred Hiatt (2008: 56), la question centrale était: «Doit-on considérer que les Antipodéens sont l'image-miroir des hommes du monde connu, ou qu'ils sont des monstres ne descendant pas d'Adam? "Saint Augustin comme Isidore voyaient dans les Antipodéens des races monstrueuses, des créatures difformes, des bêtes fabuleuses. Saint Augustin en particulier refusait la possibilité d'une présence humaine au-delà de l'œkumène: puisque la parole de Dieu ne pouvait les atteindre, les Antipodéens ne pouvaient être humains. Mais ce n'était pas là une opinion partagée par tous, et le débat continua. Hiatt (ibid.: 58) fait la remarque suivante: «Cette question agita tout le Moyen Âge et devint particulièrement sensible au moment de la découverte du Nouveau Monde: la présence de vastes populations jusqu'alors étrangères au monde chrétien semblait jeter le doute sur la théorie d'une monogenèse qui aurait commencé avec Adam. »

La controverse de Valladolid, qui suivit les premières explorations espagnoles et portugaises dans les Amériques, fut sans nul doute l'un des moments les plus intenses de ce long débat. Elle fut initiée en 1550 par Charles Quint, roi d'Espagne, et le pape Paul III. Une armée d'éminents juristes et théologiens fut appelée à se rassembler au collège San Gregorio de Valladolid pour apporter une réponse définitive au conflit sur la nature et le rôle des Indiens d'Amérique qui faisait rage en Espagne depuis 1493 (voir Hanke 1994; Hernandez 2001; Castro 2007). Les Indiens étaient-ils des êtres rationnels, dotés d'une âme? Ou bien la capacité de devenir chrétien leur faisait-elle défaut, les empêchant dès lors de prétendre au statut d'hommes? La controverse avait pour protagonistes Bartolomé de Las Casas, un moine dominicain qui avait accompagné Christophe Colomb au cours d'un de ses derniers voyages et avait été témoin des violences de la colonisation, et Juan Ginés de Sepúlveda, figure savante importante liée au collège des Espagnols de l'université de Bologne et à la cour papale de Rome, et qui n'avait jamais mis les pieds dans le Nouveau Monde.

Las Casas prônait l'unité fondamentale de l'humanité. Selon lui, les Indiens n'étaient pas moins rationnels que les habitants de l'Ancien Monde et étaient parfaitement capables de recevoir la foi chrétienne. «lls ne sont ni ignorants, ni inhumains, ni des bêtes», insistait-il. "Les Indiens sont nos frères, et le Christ est mort pour eux. » (Hanke 1994: 76) Sepúlveda, à l'inverse, les considérait comme des barbares, comme une race inférieure d'«esclaves naturels» qui, trop souvent, cédaient à toutes sortes d'idolâtries et de pratiques répréhensibles comme le cannibalisme. Selon lui, ils n'étaient pas entièrement humains (car on ne pouvait les convertir) et tenaient davantage des monstres et bêtes des antipodes évoqués par saint Augustin et Isidore. À l'argument de l'exceptionnelle habileté artistique 
des indigènes, Sepúlveda rétorquait: "Bien qu'ils fassent parfois montre de talent dans la pratique de certains artisanats, cela ne prouve pas qu'on ait affaire à une habileté de type humain, car nous voyons des petits animaux, qu'ils soient oiseaux ou araignées, fabriquer des choses qu'aucune technique humaine n'est capable d'imiter absolument." (ibid.: 85). Je ne souhaite pas m'attacher ici à évaluer les conséquences de la controverse sur le sort des Indiens (la littérature sur le sujet est abondante). Je voudrais plutôt insister sur l'observation essentielle suivante: à l'heure des Grandes Découvertes, le «nous» œcuménique ne faisait l'objet d'aucun consensus.

Au XVIII siècle commença à se cristalliser le concept d'«espèce humaine». Dans son important traité de taxonomie, Systema naturae, le botaniste et zoologiste suédois Carl von Linné décrit les Américains comme des êtres "de la couleur du cuivre, colériques, régis par la coutume", tandis que les Européens sont "clairs, doux, inventifs, gouvernés par la loi ». Mais pour les Européens des lumières, il ne faisait plus de doute que tous appartenaient à une seule et même espèce: Homo sapiens. L'autre grand naturaliste de l'époque, le comte de Buffon, démontra que la faculté de parole était le trait commun à toute l'humanité. Dans sa monumentale Histoire naturelle, il écrit que puisque les sauvages, comme les civilisés, parlent, ils sont donc eux aussi humains. Pour lui, le vrai fossé se situe entre les hommes et les animaux non doués de parole. Dans le chapitre sur la "nature de l'homme», il insiste sur le fait qu'«il y a une distance infinie entre les facultés de l'homme et celles du plus parfait animal; preuve évidente que l'homme est d'une différente nature, que seul il fait une classe à part " (Buffon 1839: 224). Buffon admet l'existence de nombreuses ressemblances entre les hommes et les animaux (la peau et le sang, le mouvement, etc.), mais ces similitudes ne sont que superficielles et ne réduisent en aucun cas leur profonde différence. En d'autres termes, le «nous» œcuménique se limitait ici, sans ambiguïté, aux membres de l'espèce humaine.

Cette conception semble avoir été très largement admise dans l'Europe du xvIII' siècle. Mais les frontières exactes de l'humanité furent remises en question. Parmi les critiques se trouvait l'Écossais lord Monboddo qui, contre Buffon, défendait l'idée que l'orang-outan (il s'agissait chez lui d'une catégorie qui incluait non seulement les orangs-outans à proprement parler, mais aussi les chimpanzés) appartenait à l'humanité. Dans le deuxième tome de son traité sur l'origine du langage, il soutient de façon catégorique que «l'orang-outan est un animal de forme humaine, à l'intérieur comme à l'extérieur ». Selon lui, le singe est intelligent, éprouve des sentiments d'amour et d'amitié, et possède le sens de l'honneur et de la justice. En résumé, toutes les qualités humaines. Notons cependant que Monboddo ne remet pas en cause le point principal, à savoir qu'il ne conteste pas l'existence d'un fossé entre les hommes et les animaux; son seul désaccord porte sur ce que ces deux catégories recouvrent. Le XVIII siècle a sans conteste défini l'« espèce humaine» comme le «nous» œcuménique par défaut. L'ironie veut que Monboddo ne fut pas pris au sérieux par ses contemporains, alors même que ses théories se révélèrent plus «justes» que celles de Linné ou de Buffon, du moins sur un point important. Avec l'émergence de la théorie de l'évolution au xix siècle, les frontières du "nous" œcuménique s'élargirent, au-delà même de ce que Monboddo avait pu imaginer: non seulement 


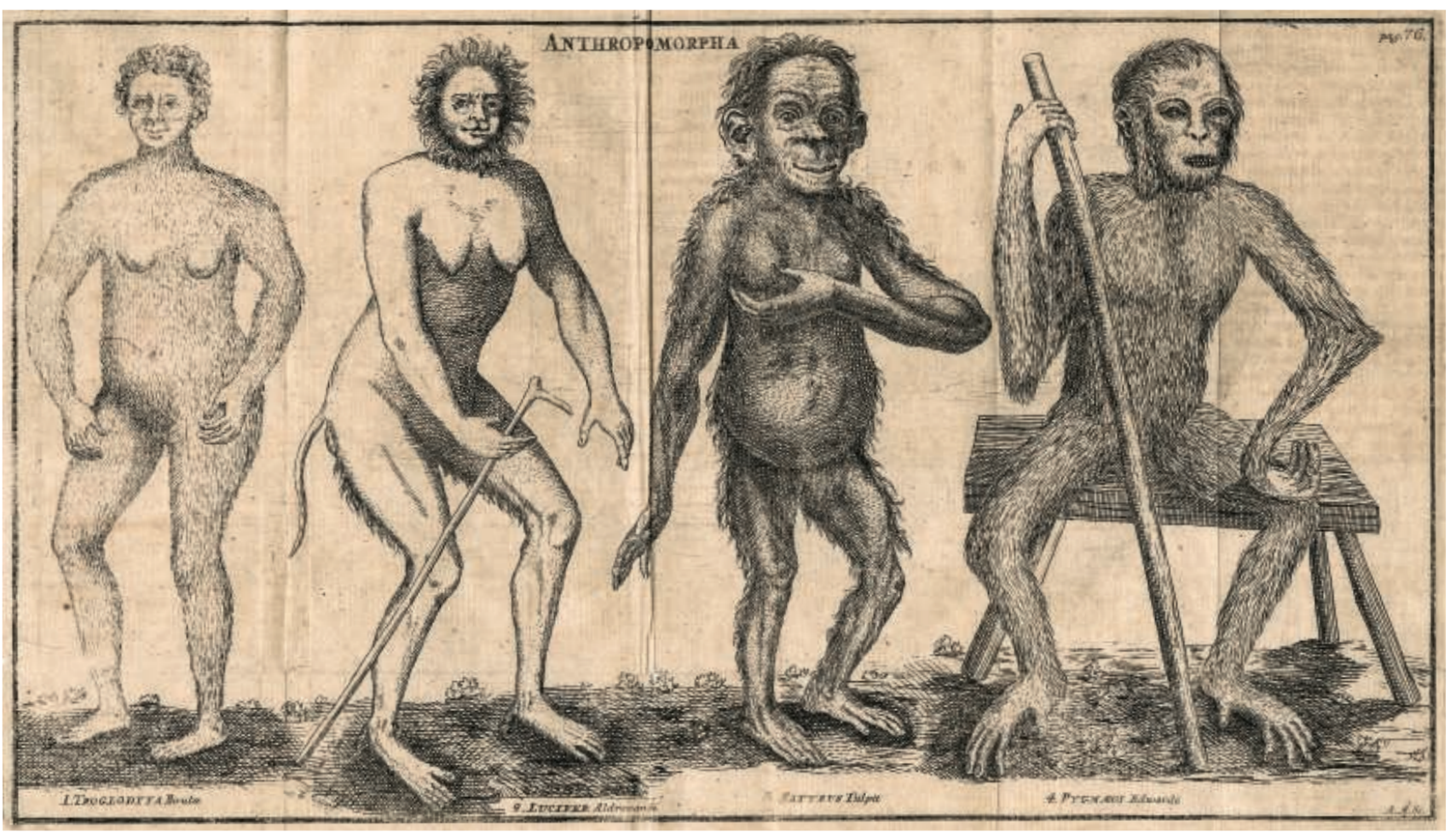

fig. 5

Quatre figures humanoïdes avec de gauche à droite: Troglodyta Bontu, Lucifer Aldrovandi, Satyrus Tulpii et Pygmaeus Edwardi in "Anthropomorpha" de Christianus Emmanue Hoppius, 1760, publié dans le volume 6 de Amoenitates academicae de Carl von Linné (Stockholm, 1763) (C) The Royal Society. 
elles incluaient désormais les grands singes, mais aussi tous les autres êtres vivants.

L'image darwinienne d'un système d'interconnexions complexes représentées par l'«arbre de la vie» joua un rôle majeur: le «nous» œcuménique dominant passa ainsi de l'espèce humaine à la vie terrestre en général. Deux siècles de biologie ont certes affiné notre compréhension de la vie, mais sans jamais produire encore de remise en question de son statut universel. Néanmoins, cette continuité apparente n'est peut-être qu'une illusion. Si on adopte une perspective historique à long terme, on se rend compte que le «nous» œcuménique a toujours fait l'objet de discussions et de débats. Au cours de l'histoire, la notion a évolué, parfois de façon soudaine et radicale. Brosser rapidement l'histoire de cette évolution d'un siècle à l'autre, comme je viens de le faire, peut être problématique à certains égards, mais cette vision globale a au moins le mérite de relativiser l'idée selon laquelle le «nous » œcuménique qui domine aujourd'hui - «la vie sur Terre» - serait maintenant établi de manière définitive. Pourquoi notre époque serait-elle différente des précédentes? Pourquoi notre «nous» occidental moderne ne susciterait-il pas à nouveau des controverses et des retournements soudains? Comment se fait-il que la grande majorité des scientifiques, et de l'opinion publique en général, soit convaincue que la vie, dans sa définition biologique actuelle, est le facteur universel ultime? J'estime que ces questions sont particulièrement pertinentes pour l'astrobiologie. Mais commençons d'abord par résumer ce que nous avons vu jusqu'à présent.

\begin{tabular}{|c|c|}
\hline Époque & «Nous œ œcuménique \\
\hline $\begin{array}{c}\text { Antiquité - Grèce ancienne } \\
\text { et monde romain }\end{array}$ & $\begin{array}{c}\text { Oikouménè, les habitants } \\
\text { du monde connu }\end{array}$ \\
\hline Moyen Âge - Occident latin & $\begin{array}{c}\text { Nous, chrétiens } \\
\text { (et ceux qui peuvent le devenir) }\end{array}$ \\
\hline Renaissance - début de l'ère moderne & Nous, membres de l'espèce humaine \\
\hline $\begin{array}{c}\text { Occident après l'émergence } \\
\text { de la théorie de l'évolution }\end{array}$ & Nous, êtres vivants \\
\hline
\end{tabular}

\section{L’astrobiologie, lieu privilégié des débats actuels autour du "nous" œcuménique}

Revenons maintenant à l'exploration de Titan. J'ai déjà mentionné la découverte récente de grands lacs composés de méthane, d'éthane et de nitrogène près des pôles de la lune de Saturne. En outre, de nombreux indices semblent suggérer l'existence d'un océan souterrain: en moins de deux ans d'intervalle, la sonde Cassini a par exemple relevé que le niveau de la surface avait augmenté de $30 \mathrm{~km}$. Cela signifie que la croûte de Titan est sans doute désolidarisée de l'intérieur et qu'il existe donc une souscouche de liquide sous la surface. Les spéculations sur la possibilité d'une vie dans ces environnements extrêmement froids, mais liquides, sont légion. Différents astrobiologistes ont essayé d'imaginer à quoi pourrait ressembler un milieu vivant où, au lieu de l'eau, ce sont les hydrocarbures qui agiraient comme solvants (par exemple Raulin 2008; Bains 2004; McKay et Smith 2005). Sans doute est-il révélateur que ces chercheurs se soient focalisés 
sur ce qu'on pourrait trouver à l'intérieur de ces corps liquides. De manière générale, on ne discute pas le statut de ces corps liquides eux-mêmes: la plupart du temps, on estime simplement que les lacs et les hypothétiques océans de Titan ne sont pas vivants puisqu'ils ne sont manifestement pas comme «nous». Mais dans quelle mesure cette non-ressemblance va-t-elle de soi? Redescendons un moment sur Terre.

Les grands corps liquides terrestres ne font généralement pas partie du «nous » œuménique: dans nos sociétés occidentales, en tout cas, il serait curieux de parler de «nous, hommes et lacs» ou «nous, hommes et océans». À la différence de la biologie, l'hydrographie et l'océanographie sont généralement perçues comme des «sciences de la troisième personne». Comme l'a montré l'un des rares anthropologues à s'être intéressé à l'astrobiologie et à l'océanographie, Helmreich (2008), dans un livre judicieusement intitulé Alien Ocean, cette mise à distance ne tombe pas sous le sens. En réalité, elle est même profondément contradictoire si on considère que toute vie sur Terre trouve son origine dans la mer et que la composition chimique des liquides du corps humain n'est pas si différente de celle de l'eau de mer. C'est ce qu'avait démontré, de façon quelque peu spectaculaire, le physicien René Quinton quand, au début $d u x^{e}$ siècle, il avait fait presque saigner à mort un berger allemand avant de remplacer le plasma sanguin par de l'eau de mer (le chien survécut, apparemment indemne). De nos jours, les équipes de secours réalisent sans attendre d'arriver à l'hôpital des transfusions de solutions salines aux victimes d'accidents (Gambino 1992: 234). Les humains (comme bien d'autres êtres vivants) sont en effet des corps liquides très semblables aux océans terrestres, abstraction faite de la différence d'échelle. Quand on se tourne maintenant vers les corps liquides de Titan, on peut se demander si, bien qu'ils soient composés d'hydrocarbures, «ils» sont tellement différents de «nous», corps aqueux. S'il est difficile de répondre à cette question, il n'en reste pas moins que l'interrogation fondamentale de l'astrobiologie, «sommes-nous seuls dans l'univers? », prend alors un tout autre sens.

Ce type de raisonnement, qui remet en question nos idées préconçues sur ce que recouvre le «nous» œcuménique, est loin d'être un cas isolé en astrobiologie. Regardons la façon dont les chercheurs conçoivent l'atmosphère de Titan. J'ai déjà dit qu'elle est principalement composée de nitrogène, tout comme l'atmosphère de la Terre, et que son système climatique repose sur un cycle de méthane analogue au cycle de l'eau sur notre planète. II y a des vents saisonniers et, au pôle sud de la lune, un ouragan permanent. D'un point de vue technique, on appelle ces tourbillons dépressionnaires des «structures dissipatives instables» (far from equilibrium dissipative structures); elles ne sont pas rares dans le système solaire. On pense par exemple aux tourbillons de poussière observés sur Mars, ou encore à la fameuse Grande Tache rouge de Jupiter. Ces structures peuvent en un sens paraître animées de vie, mais on ne les qualifie pas d'êtres vivants au sens biologique. Ce n'est pas un hasard si la météorologie, tout comme l'océanographie, est généralement perçue comme une «science de la troisième personne»: le présupposé de départ est que les nuages, la pluie, les vents et les ouragans (qu'ils soient composés d'eau ou de méthane) ne sont manifestement pas comme «nous». Pourtant, des 


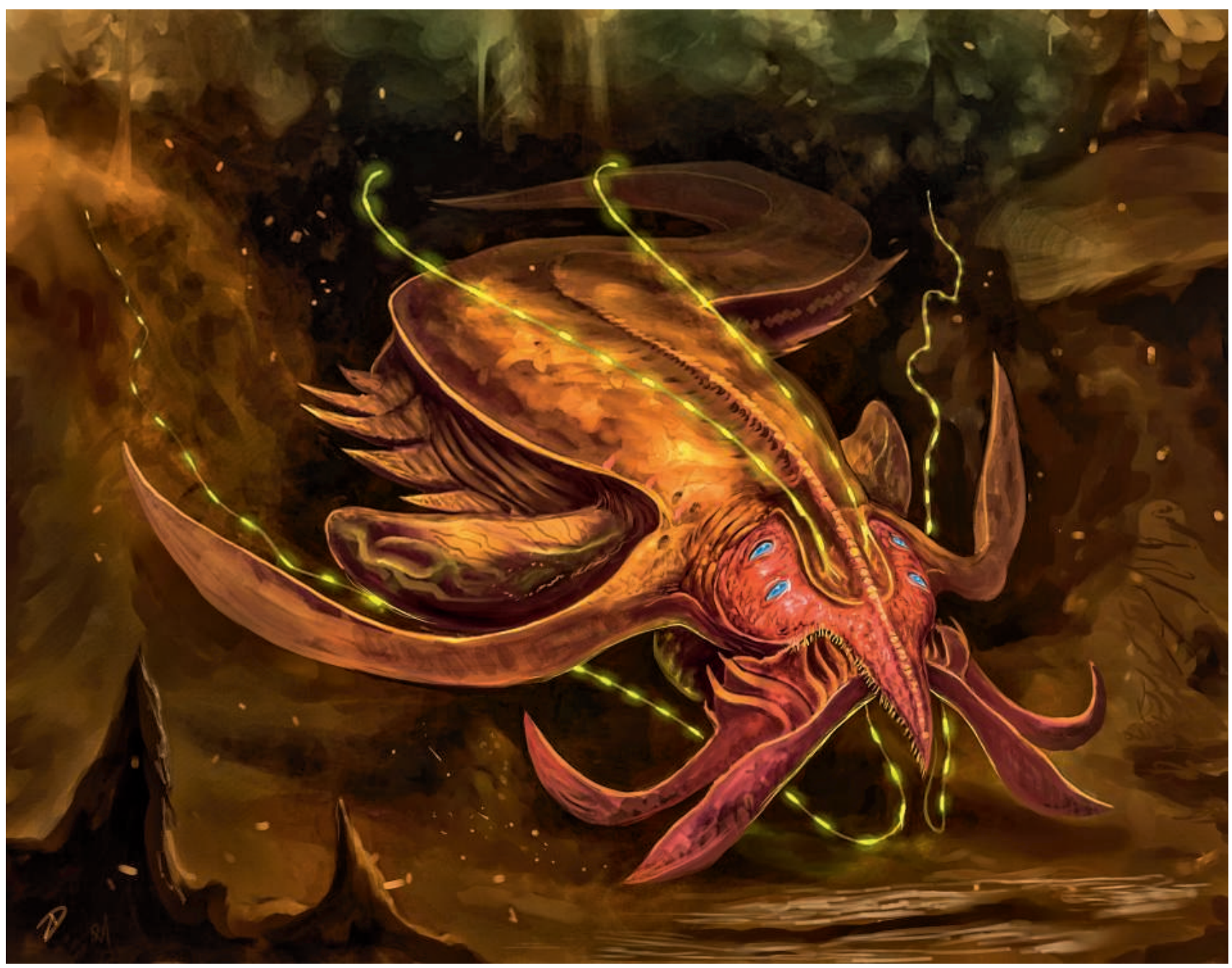

fig. 6

Dave Melvin,

Vie imaginaire dans les lacs

de Titan, 2009-2015

(c)//davesrightmind.

deviantart.com 
astrobiologistes commencent à mettre en doute l'évidence même de ces prétendues distinctions. Le planétologue Charles H. Lineweaver (2006: 7) a fait à cet égard une remarque tout à fait pertinente:

À l'équateur [de la Terre], il y a une grande diversité de formes de vie à base de carbone et davantage d'ouragans. Ceci n'est absolument pas un hasard. Les arbres de la forêt tropicale et les ouragans possèdent la même structure. II s'agit de structures dissipatives instables qui diffusent les gradients radiatifs, thermiques, chimiques et de pression créés par le rayonnement du Soleil.

Lineweaver suggère par là que «nous », êtres vivants et phénomènes météorologiques, possédons une base très semblable. Selon lui, les définitions biologiques actuelles de la vie sont trop étroites pour être utiles à une échelle cosmique. II propose une définition plus large fondée sur des principes thermodynamiques plutôt que sur le critère traditionnel de l'ADN. Si on accepte cette conception plus englobante, la conclusion est inévitablement, affirme Lineweaver, que «nous» ne sommes pas seuls: on a déjà repéré une vie extraterrestre! Tout au moins, il existe une structure dissipative très familière au pôle sud de Titan. Les différentes tentatives de Lineweaver pour que soient abandonnées les définitions biologiques classiques de la vie sont pour l'instant restées lettre morte. Mais il a au moins eu le mérite d'attirer l'attention sur le flou inhérent au «nous» dans la question centrale que se pose l'astrobiologie.

En outre, les préoccupations de Lineweaver sont partagées par bon nombre de ses collègues. Lors d'un déjeuner au colloque d'astrobiologie déjà mentionné, je me suis retrouvé entouré de scientifiques partisans d'une conception élargie du «nous». Même si la question de savoir jusqu'où l'on pouvait ou devait en repousser les limites était loin de faire consensus, mes compagnons de tablée partageaient tous l'opinion selon laquelle «les conséquences fortuites de l'évolution darwinienne sur une planète en particulier » (selon l'expression de l'un d'entre eux) n'étaient qu'une manifestation parmi d'autres d'un phénomène plus général. Ils s'accordaient également à dire que les définitions scientifiques actuelles de la vie pâtissent d'une forme de raisonnement circulaire: elles permettent certes de définir précisément ce qui est en vie, mais dans le même temps (comme le fit remarquer un autre participant) elles «limitent les réponses possibles». Un troisième ajouta: "Peut-être la définition de ce qui est vivant ou non est-elle dans le regard de celui qui observe. »

De telles réévaluations du «nous» œcuménique (à savoir que le «nous» recouvrirait avant tout les corps liquides ou les structures dissipatives plutôt que les êtres vivants) font certes l'objet de débats animés en astrobiologie, elles n'en restent pas moins très marginales dans l'opinion publique en général. L'une de ces redéfinitions est cependant parvenue à toucher le grand public. Je fais ici référence à la célèbre théorie défendue par un astrobiologiste avant la lettre, Lovelock: I'hypothèse Gaïa. Dans les années 1960, Lovelock travaillait pour la NASA où il participait à divers projets dont l'objectif était de détecter une vie extraterrestre. Il avait remarqué que Mars ne possédait pas le système autorégulateur complexe qui carac- 
térise la biosphère terrestre, et en conclut qu'il ne pouvait y avoir de vie. II proposa alors une nouvelle conception de la Terre: en tant qu'entité, elle était un être vivant. Selon lui, la circulation thermohaline de l'eau des océans équivaut à la circulation du sang chez les mammifères, les nuages n'étant pas de simples phénomènes chimiques et atmosphériques mais devant plutôt être rapprochés des poils ou de la fourrure (voir Harding 2006: 129 et 138). Comme on peut s'en douter, l'idée provoqua de nombreux débats (qui continuent à ce jour). Bien des scientifiques ont été scandalisés par l'idée qu'une planète entière soit vivante, d'autres ont au contraire pris fait et cause pour cette théorie. Mais mon but n'est pas ici d'apporter ma pierre au débat.

Je préfère au contraire montrer qu'on peut replacer la question dans un cadre beaucoup moins polémique. Au début du $x^{e}$ siècle, le géochimiste russe Vladimir Vernadsky fut le premier à explorer la possibilité d'une autre base au «nous » œuménique. Pour le dire rapidement, le «nous» recouvrait selon lui les êtres minéraux ou géologiques; la vie, c'était un type de roche particulier. II avait en effet compris que les êtres vivants, considérés en agrégat, constituent l'une des principales forces géochimiques sur Terre; des plus petits vers et algues aux plus grands oiseaux et mammifères, tous produisent, transportent et transforment quelque cinquante minéraux différents à travers le monde entier. Dans cette perspective, un troupeau d'oies en migration représente un système géologique transportant du nitrogène, tandis qu'un essaim de termites (dont les corps produisent de la magnétite) est un mécanisme de redistribution de fer (Vernardsky 1998 [1926]: 62-63). On peut également voir les hommes, qui sont (entre autres) composés de calcium et de phosphore, comme des minéraux ou des roches en mouvement; ils sont du même ordre que les processus d'érosion ou de plaques tectoniques. L'avantage de cette représentation est que le «nous» œcuménique devient plus englobant («nous, êtres géologiques", "nous, organismes, et notre planète") sans qu'il soit besoin de penser la Terre comme un être vivant, idée qui, pour une raison ou une autre, pose tant de difficultés à la plupart des chercheurs. Mais que l'on adopte la solution de Vernadsky ou celle de Lovelock, le résultat est le même: l'objet de la géologie n'est plus distinct du «nous", les sciences de la Terre deviennent des "sciences de la première personne».

Comme je l'ai déjà indiqué, on considère aujourd'hui que Titan est l'un des corps célestes qui ressemble le plus à la Terre au sein de notre système solaire. Comme la Terre, on dit qu'il est «géologiquement actif». À la différence de la plupart des autres lunes et planètes en orbite autour du Soleil, la surface de Titan est vierge de tout cratère d'impact, ce qui explique qu'on dit souvent qu'elle est «jeune». Tout comme la Terre, Titan possède une atmosphère et un système climatique complexe qui en transforme continuellement la surface. Bien que les températures y soient beaucoup plus basses, la pression atmosphérique à sa surface est remarquablement semblable à celle de la Terre. Les flux cycliques de méthane qu'on y détecte sont analogues aux cycles de l'eau sur notre planète. L'atmosphère, riche en méthane, permet de capter l'énergie libre des ultraviolets, qui pourrait servir à déclencher des réactions métaboliques; en d'autres termes, elle pourrait permettre la "photosynthèse sur une planète pauvre" (Coustenis et Blanc 2012: 194). En outre, on a trouvé sur Titan des traces de cryo- 


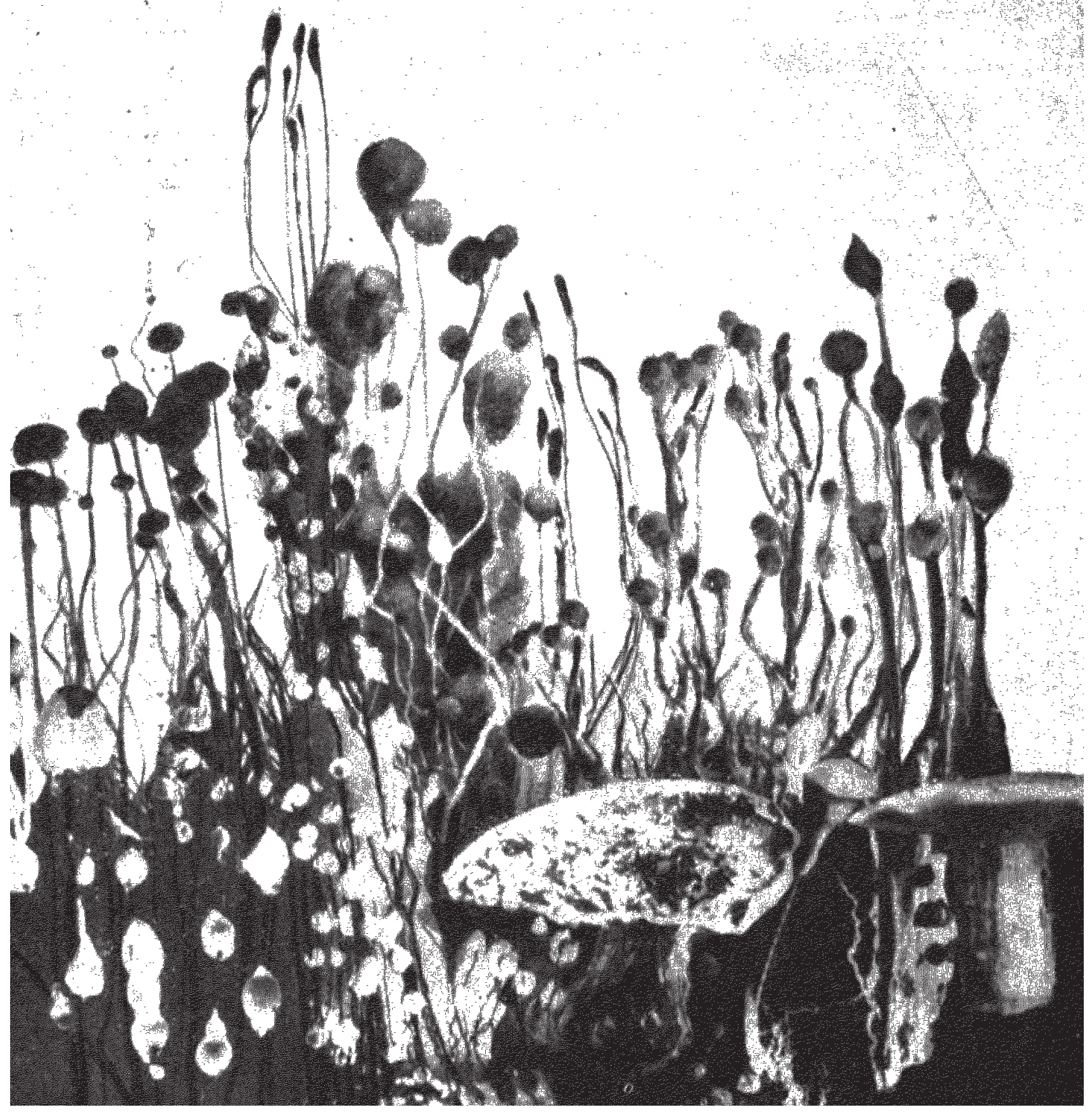


volcans: il s'agirait de volcans éjectant de la glace et/ou du méthane, et non plus de la roche en fusion. II n'est donc pas surprenant, dans ce contexte de recherche d'une vie sur Titan, que des astrobiologistes aient fait référence à l'hypothèse Gaïa. David Grinspoon, par exemple, a suggéré que, de la même manière que la Terre et ses habitants vivants avaient connu une évolution parallèle, la même chose se serait produite sur la plus grande lune de Saturne. En astrobiologie, cette extension de l'hypothèse Gaïa est connue sous le nom d'«hypothèse des mondes vivants " (Mullen 2005; Schulze-Makuck et Grinspoon 2005). Au cœur de cette théorie, on trouve l'idée selon laquelle les corps célestes qui sont «vivants » d'un point de vue géologique et météorologique ont aussi plus de chance d’héberger une vie biologique.

Un autre indice quelque peu étrange va dans le sens de l'hypothèse des mondes vivants: la présence de $5 \%$ de méthane dans l'atmosphère basse de Titan. Cette concentration relativement élevée ne s'explique pas directement par l'effet de réactions chimiques connues. Sur Terre, le méthane est avant tout le produit de l'activité microbienne. Certains défendent l'idée selon laquelle, sur Titan, la fonte massive de glace repérée à plusieurs endroits est provoquée par la chaleur rejetée par certaines formes de vie, les cryovolcans servant peut-être alors d'oasis à ces micro-organismes. ॥ est bien entendu que, pour l'instant, ce ne sont là que conjectures. II est en revanche certain que la surface de Titan est avant tout composée d'eau (sous forme de glace), à l'image de la Terre et des organismes producteurs de minéraux qui y vivent. En principe, on ne peut donc rejeter l'existence d'un «nous» œcuménique qui comprendrait la lune géante de Saturne, notre planète et tous les êtres vivants terrestres. Il est sans doute prématuré de parler de «nous, êtres vivants» (c'est-à-dire la lune, la planète et les organismes biologiques) étant donné notre connaissance actuelle limitée de la physiologie de Titan. Mais on peut véritablement s'autoriser à parler d'un dénominateur commun. On peut dire que Titan, la Terre et les êtres vivants terrestres (c'est-à-dire des minéraux qui marchent, rampent et volent, dans le sens que leur donne Vernardsky) sont «nous, êtres géologiquement actifs » ou «nous, êtres rocheux». Ces théories astrobiologiques montrent que la planétologie n'a pas à se confiner à la «science de la troisième personne» que l'on voit d'habitude en elle.

II peut paraître un peu tiré par les cheveux, à première vue, de regrouper les hommes et une lune du système solaire externe; mais en astrobiologie, où l'accent est sans cesse mis sur le lien étroit entre les êtres vivants et l'environnement stellaire, un tel rapprochement n'est pas si surprenant que cela. II est peut-être bon de se souvenir également que, comme les anthropologues et Lévi-Strauss en particulier l'ont souvent souligné, il était impensable, il y a quelques siècles à peine, de vouloir ranger dans la même catégorie des chrétiens d'Europe et des Indiens d'Amérique. De plus, Titan n'est pas l'unique exemple d'un corps céleste ressemblant de façon presque troublante à la Terre. Des recherches spatiales récentes ont confirmé que les principaux éléments participant à la fabrication de la vie - l'hydrogène, le carbone, l'oxygène et le nitrogène - sont également présents dans les comètes. Dans leur livre Où sont les autres?, les astrobiologistes Dominique Proust et Jean Schneider (2007: 94) comparent de

\section{ci-contre}

fig 7

Végétation osmotique in Stéphane Leduc, Théorie physico-chimique de la vie et générations spontanées. Paris, Poinat, 1910, fig. 62 p. 163 (C) Photo D.R. 


\section{ci-contre}

fig. 8

Ruben Ochoa, Zoned Out in the 90045, 2007.

Avec l'aimable autorisation de l'artiste et de Susanne

Vielmetter, Los Angeles

Projects. façon éclairante la composition du Soleil, celle d'une célèbre comète et celle du corps humain.

\begin{tabular}{|c|c|c|c|}
\hline Élément & Soleil & Comète de Halley & Homme \\
\hline Carbone & $0,02 \%$ & $9,5 \%$ & $11 \%$ \\
\hline Hydrogène & $92 \%$ & $63 \%$ & $55 \%$ \\
\hline Oxygène & $0,08 \%$ & $26 \%$ & $28 \%$ \\
\hline Nitrogène & $0,01 \%$ & $1 \%$ & $2 \%$ \\
\hline
\end{tabular}

Proust et Schneider démontrent ainsi qu'il n'est pas si absurde que cela de parler de "nous, hommes et comètes", en opposition avec des étoiles comme le Soleil. Dans cette perspective peu commune, "nous" se retrouve partout, non seulement sur la Terre mais dans tout le système solaire. Comme bien d'autres de leurs collègues, ces chercheurs mettent indirectement en doute le «nous" œcuménique communément accepté par tous depuis le développement de la biologie moderne. Leurs observations suggèrent que l'histoire aurait pu être bien différente. Imaginons une situation dans laquelle la grande majorité des scientifiques occidentaux se seraient entichés de Vernadsky plutôt que de Darwin. Alors les scientifiques n'auraient peut-être pas toujours répondu par la positive à la question "sommes-nous seuls dans l'univers?».

\section{Conclusion}

À partir du cas de Titan, j'ai esquissé dans cet article les contours d'une approche tentant de relier l'anthropologie et l'astrobiologie. II est pour cela essentiel de mieux comprendre ce que j'ai évoqué ici sous le nom de «nous» œcuménique. L'anthropologie, qui s'intéresse depuis longtemps à l'étude des frontières entre les différents "nous» et «les autres», est sans nul doute l'une des disciplines les mieux armées pour aborder la question en détail (même si l'histoire et la philosophie des sciences pourraient également y prétendre). Nous avons montré, quoique de manière un peu rapide et générale, que le «nous» œcuménique actuellement dominant en Occident - la vie terrestre - possède sa propre histoire, elle-même pleine de détours et de rebondissements. Depuis le développement de la biologie moderne, la notion de "nous, êtres vivants" comme «nous» le plus englobant semble relativement stable. Elle a été redéfinie, certes, mais pas fondamentalement transformée. Cependant, dès qu'on adopte une perspective à plus long terme, on se rend compte que cette stabilité n'est qu'illusoire ou tout au moins éphémère. II n'y a pas si longtemps encore, on n'excluait pas seulement les orangs-outans du «nous» œcuménique, mais aussi les indigènes vivants sur le continent américain.

Le champ de l'astrobiologie est à cet égard le lieu où peuvent s'élaborer de nouvelles hypothèses stimulantes. Comme nous l'avons montré, cela peut aller du «nous, êtres liquides " (parmi lesquels les hommes, les océans terrestres et les mers de méthane du système solaire externe), ou «nous, structures dissipatives instables" (hommes, forêts humides et ouragans extraterrestres), au «nous, êtres rocheux» (hommes, planète Terre et Titan). Cette liste est loin d'être exhaustive, la littérature ethnographique sur ces nouvelles pistes restant à écrire. Ce qui unit la plupart des astrobiologistes, 


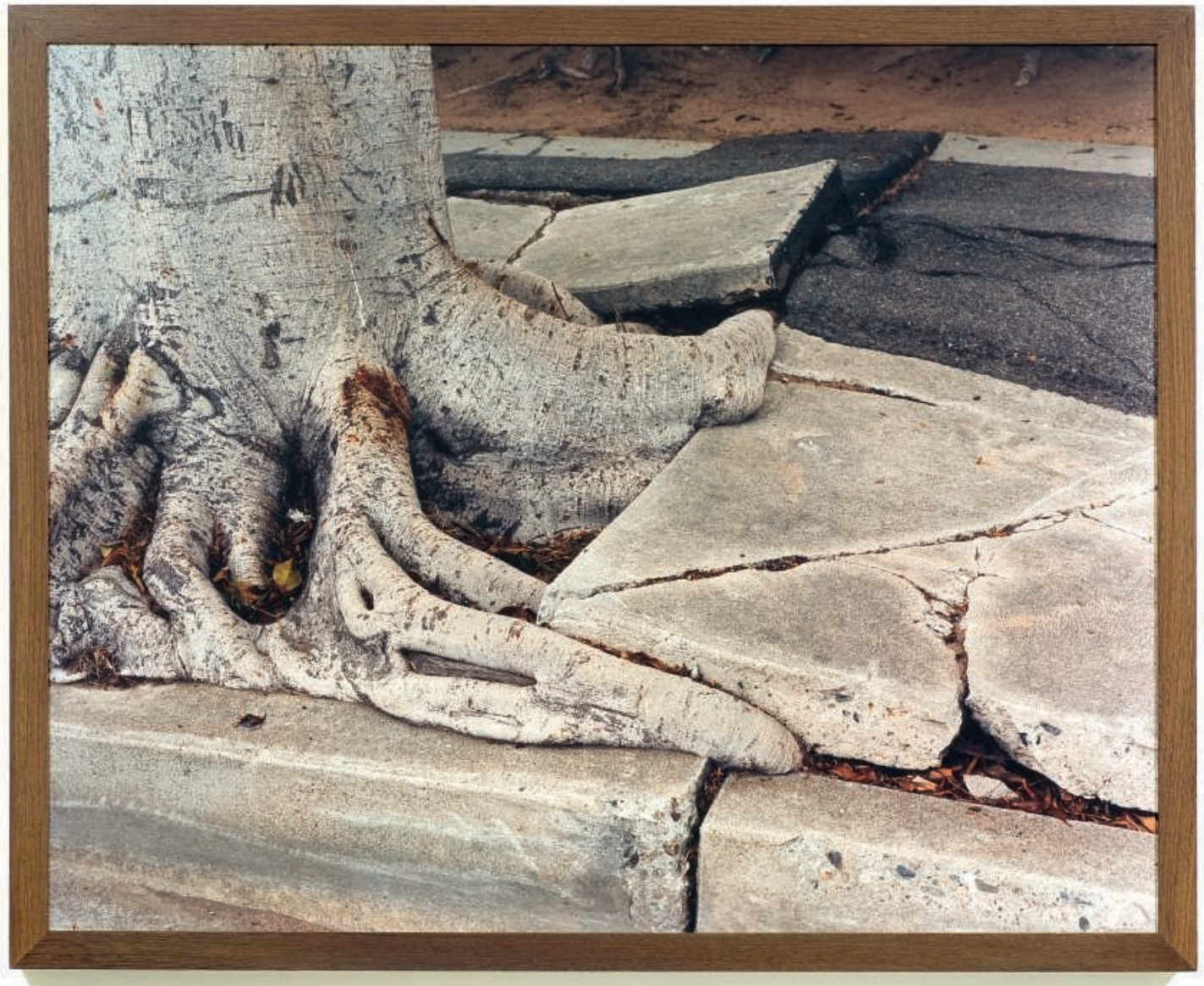


c'est leur capacité à repenser ce qui constitue «notre» plus petit dénominateur commun. Ils osent mettre en doute la croyance répandue chez les biologistes spécialistes de la Terre (ainsi que dans l'opinion publique en général) qu'il s'agit d'«être vivant », plutôt que d'«être un minéral » ou d'«être actif géologiquement». Si on choisit cette dernière catégorie, alors nous, Terriens, ne sommes manifestement pas «seuls » dans le système solaire. II est certain qu'un grand nombre de scientifiques (dont les astrobiologistes) n'y accordent aucun crédit car ce n'est là, selon eux, qu'un simple jeu de sémantique. Mon objectif n'était pas ici de prendre parti.

J'ai avant tout voulu souligner la ressemblance frappante entre ces débats contemporains et les controverses plus anciennes, telle celle de Valladolid $\mathrm{au} \times \mathrm{xl}^{\mathrm{e}}$ siècle. D'un côté, on trouve ceux qui pensent qu'un objet - la plus grande lune de Saturne ou autre - n'est évidemment pas comme «nous». De l'autre, il y a ceux qui estiment au contraire que Titan, qui est principalement composé d'eau (de glace) et de roche, est manifestement comme «nous » puisque notre corps est pour l'essentiel fait d'eau et de minéraux. Le parallèle avec le débat sur l'humanité des habitants du Nouveau Monde est si frappant qu'on peut se demander si on a affaire ici à un motif historiquement invariable ou à un aspect structurel de l'esprit moderne. Quoi qu'il en soit, Michel Morange (2009: 180) a raison quand il remarque que, dans un futur relativement proche, les astrobiologistes seront nécessairement confrontés à des objets situés à la frontière du vivant et du non-vivant: «Les qualifier d'êtres vivants ou non, ce sera là une décision humaine. »

En rapprochant l'anthropologie et l'astrobiologie, on constate une dimension spécifique de la division du travail au sein de la science occidentale moderne: «Pourquoi la curiosité emprunte-t-elle certaines voies et pas d'autres? » s'interrogent Lorraine Daston et Katharine Park (2010: 10) dans leur étude sur le merveilleux du Moyen Âge aux lumières. L'idée défendue par les deux auteures selon laquelle on devrait autant étudier les pistes écartées par les intellectuels que celles qu'ils finissent par emprunter semble tout à fait pertinente par rapport à la question «sommes-nous seuls dans l'univers? ». Comment se fait-il que ceux qui étudient l'extra-terrestre soient à ce point obsédés par l'idée de trouver d'«autres êtres vivants» plutôt que d'«autres êtres liquides» ou «êtres rocheux»? Si l'on observe la trajectoire quelque peu mouvementée du «nous» œcuménique dans l'histoire de l'Europe occidentale, la réponse est loin d'aller de soi. Ce constat ouvre un champ encore vierge à d'autres enquêtes ethnographiques. Les échelles des objets étudiés, en particulier, ont été laissées de côté. Cela apparaît immédiatement dans ce qui suit.

Le tableau ci-contre met en lumière la distinction entre ce que j'ai désigné sous le terme de «sciences de la première personne » et «sciences de la troisième personne». L'anthropologie et la biologie représentent les premières par excellence: elles se focalisent respectivement sur «nous, êtres humains» et sur «nous, êtres vivants». À l'intérieur même de la biologie, cependant, l'étude de phénomènes de grande ampleur tels que les essaims, les troupeaux ou les écosystèmes est plutôt l'exception. Quel que soit le contexte, il est curieux de parler de «nous, hommes et savanes», par exemple. Dans les sciences de la Terre et en astronomie, on trouve aussi 


\begin{tabular}{|c|c|c|c|}
\hline Discipline scientifique & Objets d'étude typiques & Échelle & «Nous» œcuménique usuel \\
\hline Astronomie & Étoiles, planètes, lunes & Très grande & Non \\
\hline Sciences de la Terre & $\begin{array}{c}\text { Océans, volcans, } \\
\text { plaques tectoniques }\end{array}$ & Grande \\
\hline Biologie & Microbes, plantes, animaux & $\begin{array}{c}\text { Du microbien à la taille } \\
\text { d'une baleine }\end{array}$ & Oui: «nous, êtres vivants» \\
\hline Anthropologie & Hommes & Humaine & Oui: «nous, hommes» \\
\hline Robotique & Sonde Cassini-Huygens & Approximativement humaine & Ambigu \\
\hline Chimie & Molécules, atomes & Petite & Non \\
\hline Physique & Particules élémentaires & Très petite & Non \\
\hline
\end{tabular}

ce genre de phénomène: prenons par exemple «nous, hommes et volcans" ou «nous, hommes et galaxies». Une grande différence d'échelle semble ainsi empêcher le déploiement d'un «nous» œcuménique. Et cela est vrai non seulement quand on atteint des échelles très grandes, mais aussi très petites. La physique et la chimie, qui sont les grandes sciences de l'infiniment petit, sont également des sciences de la troisième personne. On ne parle pas de «nous, humains et quarks» ou «nous, humains et sels». De manière générale, la règle veut que, plus on a affaire à un objet petit, moins il risque de pouvoir rentrer dans le «nous» œcuménique. L'ambiguïté de la robotique est à ce titre intéressante: selon l'endroit où l'on place la frontière (si elle existe) entre la vie «naturelle" et la vie «artificielle», il semble aller de soi ou au contraire être totalement interdit de regrouper les hommes et les robots sous un seul «nous». La grande originalité de l'astrobiologie, qui s'inspire de toutes ces disciplines, est de dépasser ces conventions. Elle est un antidote à l'autocentrisme omniprésent qui consiste à privilégier l'échelle humaine. L'astrobiologie ne part pas du présupposé que tout ce qui est compris dans le «nous» est obligatoirement de taille humaine ou presque.

$\mathrm{Au} \mathrm{xx}^{\mathrm{e}}$ siècle, les anthropologues ont joué un rôle fondamental dans l'évacuation de concepts bien connus: racisme, ethnocentrisme, sexisme... À la lumière de ce qui vient d'être exposé, on peut se demander si les astrobiologistes ne sont pas en train de faire la même chose pour un autre concept, celui-là plus inattendu: celui d'échelle. La question centrale de l'astrobiologie n'est peut-être pas tant "sommes-nous seuls dans l'univers? ", que celle-ci: "Quand un objet d'étude devient très grand, ou très petit, cela veut-il dire qu'il devient aussi de plus en plus étranger? »

Roehampton University, Londres Centre for Research in Evolutionary and Environmental Anthropology istvan.praet@roehampton.ac.uk 


\section{Bains, William}

2004 "Many chemistries could be used to build living systems", Astrobiology 4 : 137-167.

Barnes, Jason W., Brown, Robert H., Soderblom, Jason M. et al.

$\mathbf{2 0 0 9}$ "Shoreline features of Titan Ontario Lacus from Cassini/VIMS observations ", Icarus 201: 217-225.

\section{Battaglia, Debbora}

2005 E.T. Culture. Anthropology in Outerspaces. Durham et Londres, Duke University Press.

\section{Buffon, Comte de}

1839 CEuvres complètes, tome III. Paris, édition Bazouge-Pigoreau.

\section{Castro, Daniel}

\section{Another Face of Empire.} Bartolomé de Las Casas, Indigenous Rights, and Ecclesiastical Imperialism. Durham et Londres, Duke University Press.

\section{Coustenis, Athena et Blanc, Michel}

2012 "Large habitable moons. Titan and Europa ", in Chris Impey, Jonathan Lunine et José Funes (éd.), Frontiers of Astrobiology. Cambridge, Cambridge University Press.

\section{Coustenis, Athena} et Taylor, Fredric W.

2008 Titan: Exploring an Earthlike World. Singapour, World Scientific.

\section{Daston, Lorraine et Park, Katherine}

2010 Wonders and the Order of Nature, 1150-1750. New York, Zone Books.

\section{Descola, Philippe}

2005 Par-delà nature et culture. Paris, Gallimard.

\section{Dick, Steven J.}

1998 Life on Other Worlds. The 20th-Century Extraterrestrial Life Debate. Cambridge, Cambridge University Press.

Fortes, Andrew D. et al. 2000 «Ammonium sulphate on Titan: Possible origin and role in cryovolcanism ", Icarus 188: 139-153.

\section{Gambino, Henry J.}

1992 Modern Esthetics: A Scientific Source for Estheticians. New York, Milady.

\section{Gilmour, lain} et Sephton, Mark A.

2004 An Introduction to

Astrobiology. Cambridge et Milton Keynes, Cambridge University Press-The Open University.

\section{Hanke, Lewis}

1994 All Mankind is One. A Study of the Disputation between Bartolomé de Las Casas and Juan Ginés de Sepúlveda on the Religious and Intellectual Capacity of the American Indians. DeKalb, Northern Illinois University Press.

\section{Harding, Stephan}

2006 Animate Earth: Science, Intuition and Gaia. Totnes, Green Books.

\section{Helmreich, Stefan}

2008 Alien Ocean: Anthropological Voyages in Microbial Seas. Berkeley, University of California Press.

2011 "What was life? Answers from three limit biologies ", Critical Inquiry 37(4) : 671-696.

\section{Hernandez, Bonar Ludwig}

2001 «The Las Casas-Sepúlveda controversy: 1550-1551 », Ex Post Facto 10(1): 95-105.

\section{Hiatt, Alfred}

2008 Terra Incognita. Mapping

the Antipodes before 1600.

Londres, The British Library.

\author{
Huygens, Christiaan \\ 1698 Cosmotheoros. La Haye, \\ Adriaan Moetjens.
}

\section{Ingold, Tim} et Pálsson, Gísli

2013 Biosocial Becomings.

Integrating Social and Biological Anthropology. Cambridge,

Cambridge University Press.

\section{Lévi-Strauss, Claude}

1955 Tristes Tropiques. Paris, Plon.

\section{Lineweaver, Charles H.}

2006 "We have not detected extra-terrestrial life. Or have we?", in Joseph Sekbach (éd.) Life as We Know It. Dordrecht, Springer: 445-457.

\section{Lorenz, Ralph D. et al.}

2008 "Titan's rotation reveals an internal ocean and changing zonal winds", Science 319: 1649-1651.

\section{Lorenz, Ralph D.} et Mitton, Jacqueline

2008 Titan Unveiled. Saturn's Mysterious Moon Explored. Princeton, Princeton University Press.

\section{Lovelock, James}

2000 Gaia: A New Look at Life on Earth. Oxford, Oxford University Press.

\section{McKay, Christopher P.} et Smith, Heather D.

\section{5 «Possibilities for}

methanogenic life in liquid methane on the surface of Titan ", Icarus 178 274-276.

\section{Morange, Michel}

2009 Explaining Life. New Haven, Yale University Press.

\section{Mullen, Leslie}

2005 "The living worlds" Astrobiology Magazine [En ligne] ; http://www.astrobio.net/ interview/1720/the-living-worldshypothesis.

\section{Pálsson, Gísli}

2009 "Celestial bodies: Lucy in the sky ", Studies in Space Policy $1: 69-81$.

\section{Proust, Dominique} et Schneider, Jean

$\mathbf{2 0 0 7}$ Où sont les autres? À la recherche de la vie dans I'univers. Paris, Seuil.

\section{Raulin, François}

2008 "Astrobiology and the habitability of Titan ", in Oliver Botta et al. (éd.), Strategies of Life Detection. New York, Springer: 37-48.

\section{Schulze-Makuch, Dirk et Grinspoon, David H.}

2005 "Biologically enhanced energy and carbon cycling on Titan? ", Astrobiology 5(4) : 560-564

Stofan, Ellen R. et al. 2007 "The lakes of Titan", Nature 445: 61-64.

\section{Vernadsky, Vladimir 1998 [1926] The Biosphere.} New York, Copernicus.

\section{Viveiros de Castro, Eduardo}

1998 "Cosmological deixis and Amerindian perspectivism ", Journal of the Royal Anthropological Institute 4(3) : 469-488. page 136 et ci-contre Dave Melvin, Vie imaginaire dans les lacs de Titan, 2009-2015 (c) //davesrightmind.deviantart.com 
